\title{
C-H Functionalization Strategies for the Construction of Thioethers
}

\author{
CHEN Shihao ${ }^{1}$, WANG Ming ${ }^{1}$, JIANG Xuefeng ${ }^{1,2, *}$ \\ ${ }^{1}$ Shanghai Key Laboratory of Green Chemistry and Chemical Process, School of Chemistry and Molecular Engineering, \\ East China Normal University, Shanghai 200062, P. R. China. \\ ${ }^{2}$ State Key Laboratory of Organometallic Chemistry, Shanghai Institute of Organic Chemistry, Chinese Academy of Sciences, \\ Shanghai 200032, P. R. China.
}

\begin{abstract}
Thioesters, which are essential sulfur-containing organic molecules, are indispensable in natural products, pharmaceuticals, and organic light-emitting materials. Efficient synthesis of thioethers has received considerable attention due to the widespread applications of these compounds, and

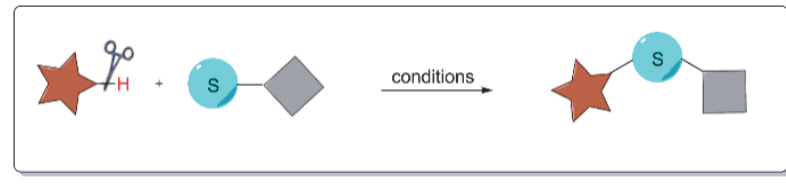
many fundamental approaches for $\mathrm{C}-\mathrm{S}$ bond formation have been proposed. However, most of them construct $\mathrm{C}-\mathrm{S}$ bonds by employing organic halides/organic boronic acid. These methodologies generally suffer from a pre-functionalized starting material. Recently, selective $\mathrm{C}-\mathrm{H}$ functionalization emerged as a powerful tool for the synthesis of $\mathrm{C}-\mathrm{N}, \mathrm{C}-\mathrm{O}$, $\mathrm{C}-\mathrm{C}$, and $\mathrm{C}-$ halogen bonds. Nevertheless, $\mathrm{C}-\mathrm{S}$ bond formation via $\mathrm{C}-\mathrm{H}$ functionalization has only recently been given more importance because organosulfur compounds are believed to inactivate catalysts. In contrast to traditional crosscoupling reactions, direct functionalization of $\mathrm{C}-\mathrm{H}$ bonds for the synthesis of thioethers can shorten the reaction steps and minimize the amount of waste formed. In this review, which is divided into several parts, we describe $\mathrm{C}-\mathrm{H}$ functionalization strategies for the construction of thioethers. In Part I, we introduce the importance and widespread applications of thioethers in daily life. For example, Lissoclibadin 6 is a polysulfur aromatic alkaloid that shows antimicrobial activity. Seroquel is an antipsychotic medicine. It is used to treat bipolar disorder and schizophrenia in adults, and children who are at least 10 years old. Tazarotene is approved for the treatment of psoriasis, acne, and sun-damaged skin. Furthermore, a comparison between conventional synthesis methods and $\mathrm{C}-\mathrm{H}$ thiolation is discussed. In Part II, we introduce copper-catalyzed or copper-mediated $\mathrm{C}-\mathrm{H}$ thiolation. Along with the direct functionalization of $s p^{2}$ and $s p \mathrm{C}-\mathrm{H}$ for the synthesis of aryl sulfides, some significant and challenging thiolations of $s p^{3} \mathrm{C}-\mathrm{H}$ are included. In addition to copper, palladium is an excellent catalyst for $\mathrm{C}-\mathrm{H}$ functionalization. In Part III, we elucidate palladium-catalyzed $\mathrm{C}-\mathrm{H}$ thiolation and discuss many proposed mechanisms. Nickel, which is a first-flow, low-cost, and earth-abundant metal catalyst, has increasingly gained attention. In contrast to copper and palladium, despite its late start, several remarkable reports on nickel-catalyzed $\mathrm{C}-\mathrm{H}$ thiolation were published by several groups. Rhodium plays a key role in selective $\mathrm{C}-\mathrm{H}$ functionalization. Some published results proved the capacity of rhodium catalysts to promote $\mathrm{C}-\mathrm{S}$ construction via $\mathrm{C}-\mathrm{H}$ functionalization. In Part IV, we introduce rhodium-catalyzed $\mathrm{C}-\mathrm{H}$ thiolation. In recent years, metal-free $\mathrm{C}-\mathrm{H}$ functionalization has been quite attractive. In Part $\mathrm{V}$, some $\mathrm{C}-\mathrm{S}$ construction strategies via metal-free $\mathrm{C}-\mathrm{H}$ functionalization are presented. In the last part, the conclusion discusses the limitations and possible development directions of these advances in the construction of thioethers.
\end{abstract}

Key Words: Thioethers; $\mathrm{C}-\mathrm{H}$ functionalization; Transition metal; Transition metal-free catalysis; Sulfur-containing compounds

Received: October 22, 2018; Revised: November 26, 2018; Accepted: November 26, 2018; Published online: November $29,2018$.

*Corresponding author. Email: xfjiang@chem.ecnu.edu.cn.

The project was supported by the National Key Research and Development Program of China (2017YFD0200500), National Natural Science Foundation of China (21722202, 21672069, 21472050, 21502054), S\&TCSM of Shanghai, China (18JC1415600), and National Program for Support of Top-notch Young Professionals, China.

国家重点研发计划(2017YFD0200500), 国家自然科学基金(21722202, 21672069, 21472050, 21502054), 上海市基础研究领域项目(18JC1415600), 中 组部 “万人计划” 青年拔尖人才资助

(C) Editorial office of Acta Physico-Chimica Sinica 


\title{
$\mathbf{C}-\mathbf{H}$ 官能化构建硫醚
}

\author{
陈世豪 ${ }^{1}$, 王明 1 , 姜雪峰 1,2 , \\ 1 华东师范大学化学与分子工程学院, 上海市绿色化学与化工过程绿色化重点实验室, 上海 200062 \\ 2 中国科学院上海有机化学研究所, 金属有机化学国家重点实验室, 上海 200032
}

\begin{abstract}
摘要: 硫醚作为一类重要的含硫功能分子, 广泛存在于天然产物、药物及有机发光材料中。鉴于硫醚类化合物的重要性, 近 年来化学家们发展了一系列高效构建硫醚的方法。与传统的有机卤化物/有机嗍酸与硫醇交叉偶联的合成方法相比, $\mathrm{C}-\mathrm{H}$ 官能化直接构建硫醚的策略因其步骤经济性、原子经济性备受合成化学家们关注, 并取得重要进展。本文根据不同过渡 金属进行分类, 系统阐述了近年来过渡金属催化/参与 $\mathrm{C}-\mathrm{H}$ 官能化或无过渡金属催化 $\mathrm{C}-\mathrm{H}$ 官能化构建硫醚这一方向研究 进展。
\end{abstract}

关键词：硫醚； C-H官能化；过渡金属；无过渡金属催化；含硫化合物 中图分类号: 0643

\section{1 引言}

硫醚是一类具有多样的生理活性 ${ }^{1-4}$ 及独特物 理化学性质 5,6 的含硫化合物, 其作为一种重要的 结构单元广泛存在于天然产物 ${ }^{7-9}$ 、药物 ${ }^{1-4,10}$ 、有机 功能材料5,11-13 中, 比如, Lissoclibadin $6^{14,15}$ 是一 种具有良好抗菌活性的多硫生物碱。Seroquel作为 一种非典型抗精神病药物用于治疗精神分裂症和 治疗双相情感障碍的躁狂发作 ${ }^{16}$ 。Tazarotene是一 种治疗银屑病、痤疮的安全高效药物 ${ }^{17}$ 。此外, 研 究表明硫醚类化合物还可作为动物交流的生物 信息素 18 。

鉴于硫醚类化合物的重要应用及广阔的潜在 应用前景, 化学家们发展了一系列构建硫醚的方 法。传统构建硫醚方法可分为两种：1)硫醇或硫酚 的 $\mathrm{S}_{\mathrm{N}} 2$ 亲核取代反应; 2)过渡金属催化的硫醇与有 机卤化物或有机嗍酸的交叉偶联反应 ${ }^{19-23}$ 。尽管这 些方法合成了一系列硫醚化合物, 但其通常伴有 一些不可忽视的缺点 ${ }^{24,25}$, 比如步骤经济性、原子 经济性较差; 有机卤化物/有机金属试剂需要预先 制备等。这些缺点在一定程度上限制了这些反应 的应用。

近年来, $\mathrm{C}-\mathrm{H}$ 官能化构建硫醚得到了巨大的 发展。与传统合成方法相比, $\mathrm{C}-\mathrm{H}$ 官能化构建硫 醚常具有以下特点 ${ }^{26}: 1$ )无需预先官能化制备有机

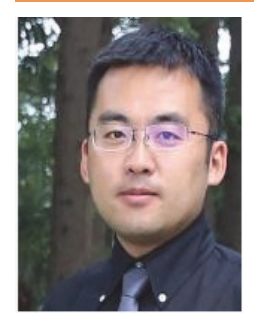

姜雪峰, 2003年获西北大学学士学

位, 2008年于中国科学院上海有机 研究所获博士学位。现任华东师范 大学教授、博导、教育部 “青年长 江学者”。研究方向为有机硫化学 和方法学导向的天然产物全合成。
卤化物或有机金属试剂; 2)具有更高的原子经济 性；3)更短的合成步骤；4)更少的废弃物生成。本 文将从不同过渡金属种类出发, 系统地阐述并总 结了近年来在过渡金属催化/参与 $\mathrm{C}-\mathrm{H}$ 官能化或 无金属催化构建硫醚这一方向的研究近况, 并对 该研究领域的局限性及发展前景进行展望。

\section{C-H官能化构建硫醚}

\section{$2.1 \mathrm{Cu}$ 催化 $/$ 参与 $\mathrm{C}-\mathrm{H}$ 官能化构建硫醚}

$\mathrm{Cu}$ 是一种良好的用于 $\mathrm{C}-\mathrm{H}$ 官能反应中构筑 $\mathrm{C}-\mathrm{S}$ 的过渡金属。2006年, 余金权课题组 ${ }^{27}$ 使用苯 硫酚或二甲基二硫醚为硫源, 以空气为氧化剂, 首 次实现了 $\mathrm{Cu}$ 参与的2-苯基吡啶的 $s p^{2} \mathrm{C}-\mathrm{H}$ 官能化 构建硫醚(图1), 作者在机理探究过程中并未发现 该反应具有动力学同位素效应, 说明该反应的反 应机制可能与其他常伴有动力学同位素效应的 $\mathrm{Pd}$ 催化 $\mathrm{C}-\mathrm{H}$ 官能化反应不同。作者认为该反应可能 经历一个单电子转移历程, 并且从吡啶铜络合物 到自由基阳离子中间体的生成为该反应决速步。

2010 年, 卿凤翎课题组 ${ }^{28}$ 同样使用吡啶为导向 基团，以廉价易得的二甲基亚砜(DMSO)和二乙基 亚砜为硫源成功实现 $\mathrm{Cu}$ 催化的2-苯基吡啶邻位 $\mathrm{C}-\mathrm{H}$ 官能化, 构建了一系列甲基硫醚及乙基硫醚。

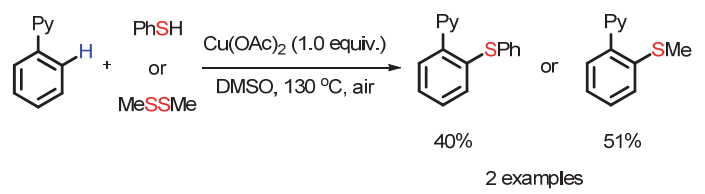

图 1 2-苯基吡啶 $\mathbf{C}-\mathbf{H}$ 官能化 ${ }^{27}$

Fig. 1 The $\mathbf{C}-\mathbf{H}$ functionalizations of 2-phenylpyridine ${ }^{27}$.

Adapted from ACS publisher. 
但需向底物中引入后期难以脱除的导向基团是这 两份工作主要的局限。

2009年, Fukuzawa等人 ${ }^{29}$ 报道了 $\mathrm{Cu}$ 催化的苯 并噁唑环的 $\mathrm{C}-\mathrm{H}$ 官能化(图2)。作者以二芳基硫醚 或芳基硫酚为硫源, $\mathrm{Cs}_{2} \mathrm{CO}_{3}$ 作碱, 氧气氛围下首 次实现了苯并噁唑环 2 位 $\mathrm{C}-\mathrm{H}$ 的硫醚化。作者认 为硫醇在体系中被氧化成二硫醚作为实际的硫醚 化试剂参与反应。遗憾的是该反应仅适用于富电 子底物, 且除了苯并噁唑之外, 啞唑、噻唑、苯并 噻唑等杂环均不能兼容。

2010年, 成江等人 ${ }^{30}$ 报道了二硫醚与负电子芳 烃通过 $\mathrm{Cu}$ 催化的 $\mathrm{C}-\mathrm{H}$ 官能化的方式构建了一系 列硫醚化合物(图3)。但该反应底物范围较窄, 仅 局限于三甲氧基苯和二甲氧基苯。

2011 年, 刘雪原等人 ${ }^{31}$ 使用 $\mathrm{Cu}(\mathrm{OAc})_{2}$ 为催化 剂, $\mathrm{CuO}$ 为添加剂以 $\mathrm{C}-\mathrm{H}$ 官能化的策略构建了一 系列杂芳环硫醚化合物。同年, 刘小刚课题组 ${ }^{32}$ 发 展的铜参与的 $\mathrm{C}-\mathrm{H}$ 活化策略成功地以良好的收 率合成了多样的杂芳环硫醚(图4)。该反应具有良 好的普适性和官能团容忍性, 烷基硫醇和芳基硫 酚以及苯并噻唑、咪唑、吲哚等杂芳环均可在标准 条件下得到相应目标产物。与前人工作相比, 作者 做了较为详尽的机理研究和密度泛函理论(DFT) 计算, 表明硫醇铜络合物为该反应首先生成的活 性中间体, 而不是通常认为的噻唑铜络合物, 而且 氧气可能参与反应协助活性位点 $\mathrm{C}-\mathrm{H}$ 断裂, 但是 该反应需要加入化学计量的铜, 且反应温度较高。

2012年, Alves等人 33 发展了 $\mathrm{Cu}$ 催化的吡咯类 杂环 $\mathrm{C}-\mathrm{H}$ 的官能化, 吡咯及其衍生物在 $\mathrm{CuI}$ 催化下 与二硫醚或硫醇反应得到一系列硫醚化合物, 该反 应只需3\%-5\%的催化剂负载量即可以中等到良好 的收率得到目标化合物。

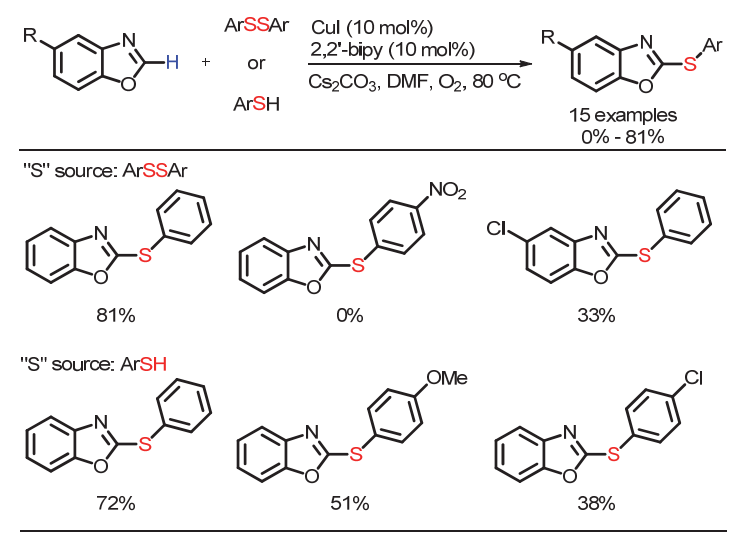

图 2 苯并噁唑 $\mathbf{C}-\mathbf{H}$ 官能化 29

Fig. 2 The $\mathbf{C}-\mathrm{H}$ functionalizations of benzoxazole ${ }^{29}$. Adapted from ScienceDirect publisher.

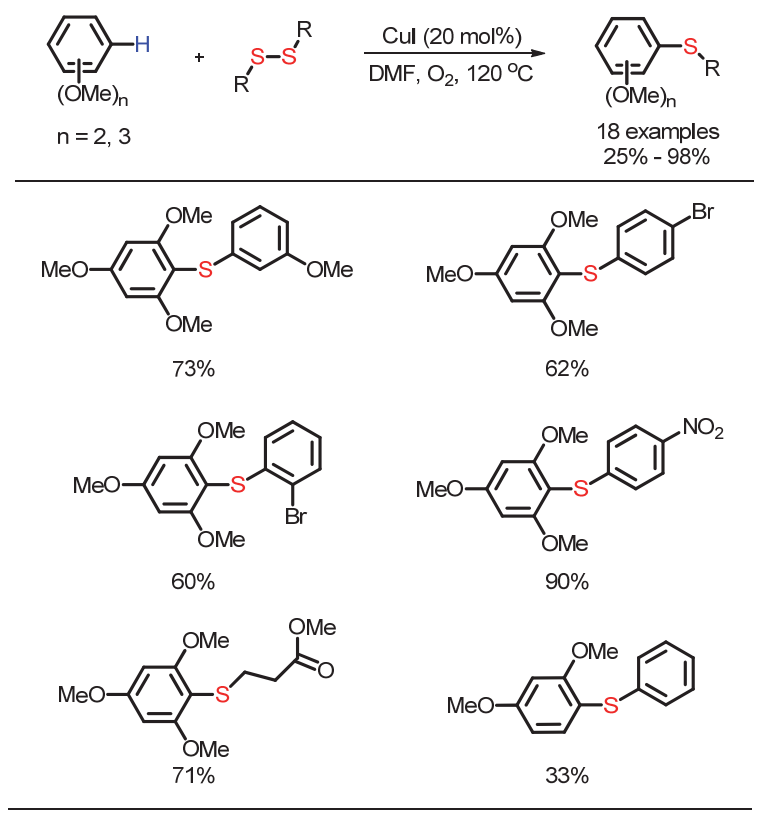

图 3 富电子芳烃 $\mathbf{C}-\mathbf{H}$ 官能化 ${ }^{30}$

Fig. 3 The $\mathrm{C}-\mathrm{H}$ functionalizations of electron-rich arene ${ }^{30}$.

Adapted from ACS publisher.

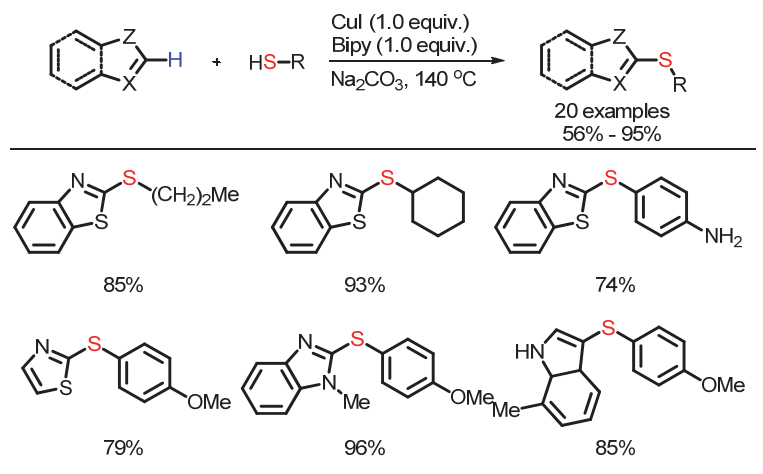

图 4 杂芳环 $\mathbf{C}-\mathbf{H}$ 官能化 ${ }^{32}$

Fig. 4 The $\mathrm{C}-\mathrm{H}$ functionalizations of aromatic heterocycles ${ }^{32}$.

Adapted from ACS publisher.

同年, 许兆青等人 ${ }^{34}$ 发展了一种Lewis酸催化, $\mathrm{Cu}$ 参与的杂芳环的 $\mathrm{C}-\mathrm{H}$ 官能化策略, 该方法以苯 并噻唑、噻唑、苯并噁唑、苯并咪唑、咪唑、噁二 唑等杂环及其衍生物为底物, 使用硫醇或芳基硫 酚实现杂芳环的 $\mathrm{C}-\mathrm{H}$ 的硫醚化反应(图5)。当使用 DMSO为溶剂时, 可以实现上述杂芳环的甲硫醚 化。在标准条件下，作者使用二硫醚为硫源，并不 能得到目标产物, 说明在该反应体系中, 硫醇并不 是生成二硫醚再参与反应。作者认为 $\mathrm{Cu}$ 在反应中 不仅作为氧化剂还可能参与形成反应中间体, 反 应可能经历以下 4 个步骤: 1)硫醇与 $\mathrm{Cu}(\mathrm{OAc})_{2}$ 形成 $\mathrm{RSCuOAc}$ 物种; 2) Lewis酸促进及羧酸根协助的金 


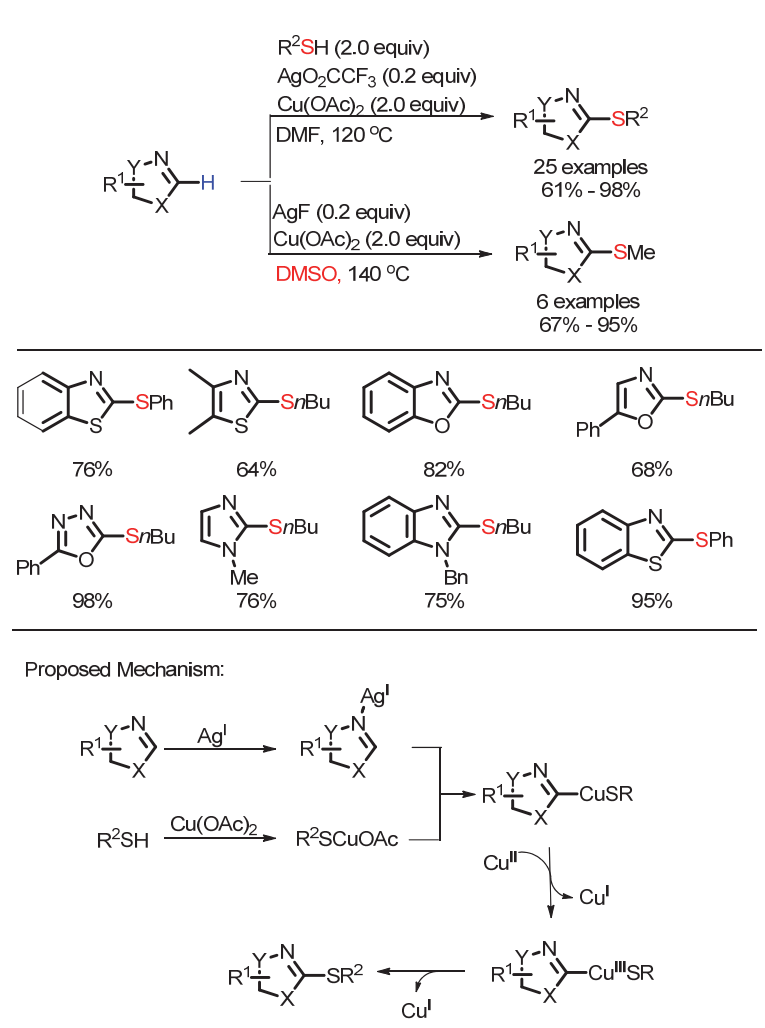

图 5 杂芳环 $\mathbf{C}-\mathbf{H}$ 硫醚化及甲硫基化 ${ }^{34}$

Fig. 5 The $\mathrm{C}-\mathrm{H}$ bond sulfenylation and methylthiolation of aromatic heterocycles ${ }^{34}$.

Adapted from ACS publisher.

属化-脱氢化生成 $\mathrm{C}_{\mathrm{Ar}} \mathrm{CuSR}$ 中间体; 3) $\mathrm{Cu}^{\mathrm{II}}$ 歧化成 $\mathrm{Cu}^{\mathrm{I}}$ 和 $\mathrm{Cu}^{\mathrm{III}}$; 4)还原消除生成产物。此外, 动力学同 位素实验说明可能的决速步是还原消除或歧化反 应而不是 $\mathrm{C}-\mathrm{H}$ 的断裂。

2013年, Zeni等人 ${ }^{35}$ 实现了纳米 $\mathrm{CuO}$ 催化的噻 唑的 $\mathrm{C}-\mathrm{H}$ 官能化, 该反应对于不同的二烷基二硫 醚和二芳基二硫醚均有较好的适用性。值得一提 的是, 当使用二硒醚时也能以中等到良好的收率 得到相应的杂芳环硒醚类产物。2015年, 曹华等 人 ${ }^{24,36}$ 同时报道了 $\mathrm{Cu}$ 催化的咪唑并 [1,2-a]吡啶的 $\mathrm{C}-\mathrm{H}$ 硫醚化反应, 该反应选择性发生在底物的 $\mathrm{C} 3$ 位。

三氟甲基芳基硫醚是药物、农药中的重要结 构单元 ${ }^{37,38}$, 由于 $-\mathrm{SCF}_{3}$ 具有强的吸电性和亲脂 性, 向分子中引入 $-\mathrm{SCF}_{3}$ 可显著改变其生理性能 和理化性质 ${ }^{39}$, 鉴于 $-\mathrm{SCF}_{3}$ 的重要意义, 化学家们 发展了一些- $\mathrm{SCF}_{3}$ 试剂 ${ }^{40-53}$, 其中许多已被应用于 $\mathrm{C}-\mathrm{H}$ 官能化构建三氟甲硫醚中。2012年, Daugulis 等人 ${ }^{54}$ 在分子中引入导向基团, 成功实现了 $\mathrm{Cu}(\mathrm{OAc})_{2}$ 促进的 $s p^{2} \mathrm{C}-\mathrm{H}$ 的硫醚化反应(图6)。作 者使用 $\mathrm{CF}_{3} \mathrm{SSCF}_{3}$ 为硫源, $\mathrm{DMSO}$ 为溶剂, 在催化量 或当量 $\mathrm{Cu}(\mathrm{OAc})_{2}$ 的促进下, 无论是富电子还是缺

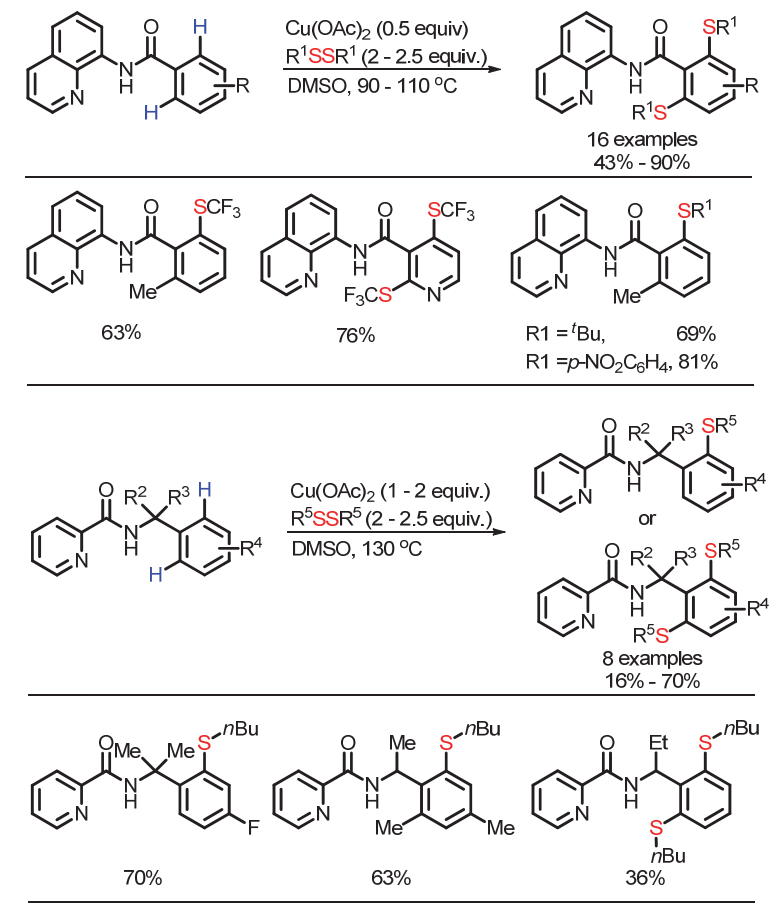

图 $6 \mathrm{Cu}$ 促进的硫醚化 ${ }^{54}$

Fig. $6 \mathrm{Cu}(\mathrm{II})$-promoted sulfenylation ${ }^{54}$.

Adapted from ACS publisher.

电子芳环, 均可以同时向分子内引入两个 $-\mathrm{SCF}_{3}$ 基团。作者还考察了二芳基硫醚和二烷基硫醚以 及不同导向基团在该策略下的适用性, 发现在标 准条件下均能得到目标产物。

2014年刘雪原等 55 同样向底物中引入 8 -氨基 喹啉作为导向基团, 实现了 $\mathrm{Cu}$ 催化的 $s p^{2} \mathrm{C}-\mathrm{H}$ 官 能化反应构建硫醚, 该反应对于 $1^{\circ} 、 2^{\circ}$ 和 $3^{\circ}$ 硫醇均 能发生反应。但是该反应存在一些局限性: 需加入 过量的 $\mathrm{Ag}_{2} \mathrm{CO}_{3}$ 为添加剂, 并且需将配体用量提升 至 $40 \%$ (摩尔分数)才能以较好收率得到目标产物。 且以上两份工作的反应机理尚不明确。

2014年, Shibahara等人 ${ }^{56}$ 使用更加廉价易得的 $\mathrm{S}_{8}$ 为硫源, 以 $\mathrm{CuTc}$ 为催化剂活化咪唑并 $[1,5-\mathrm{a}]$ 吡啶 的 $\mathrm{C}-\mathrm{H}$ 构建了一系列二芳基硫醚(图7)。除了咪唑 并[1,5-a]吡啶外, 其他富电子芳环如 $N$-甲基吲哚、 $\mathrm{N}, \mathrm{N}$-二烷基苯胺也可以发生反应。作者还将该硫 醚化反应应用于葟聚硫化物的合成。

2014年, 卿风翎课题组 ${ }^{57}$ 报道了一例 $\mathrm{Cu}$ 催化的 无导向基团的苄位 $\mathrm{C}-\mathrm{H}$ 的三氟甲硫基化反应，首 次实现了过渡金属催化的 $s p^{3} \mathrm{C}-\mathrm{H}$ 直接三氟甲硫 基化反应(图8)。在反应中, 作者使用 ${ }^{t} \mathrm{BuOOBz}(3-$ $\mathrm{CF}_{3}$ ) 为氧化剂, $\mathrm{AgSCF}_{3}$ 与 $\mathrm{KCl}$ 作为添加剂现场生 成 $\mathrm{CF}_{3} \mathrm{~S}^{-}$, 在标准条件下, 一系列甲苯衍生物可以 以中等到良好的收率得到相应的苄基三氟甲基硫 醚, 所得产物还可直接被氧化成三氟甲砜基衍生 


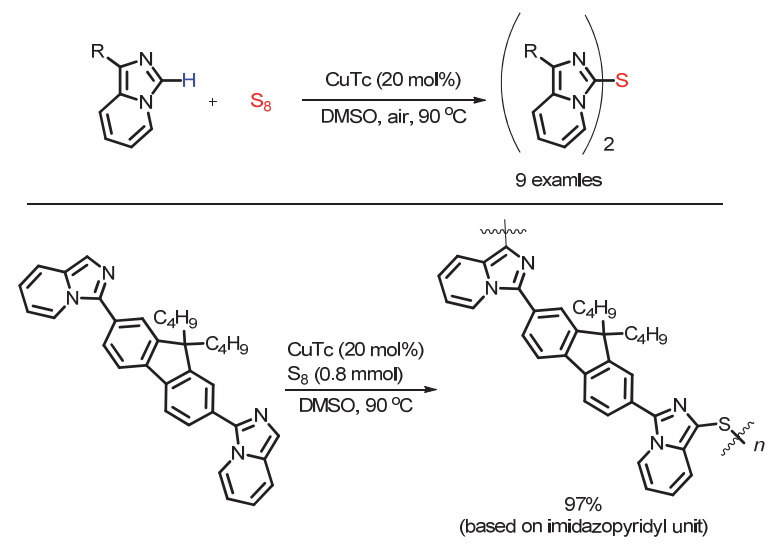

图 $7 \mathrm{~S}_{8}$ 为硫源的 $\mathbf{C}-\mathrm{H}$ 硫醚化 ${ }^{34}$

Fig. 7 The $\mathrm{C}-\mathrm{H}$ bond sulfenylation using $\mathrm{S}_{8}{ }^{34}$.

Adapted from Wiley publisher.
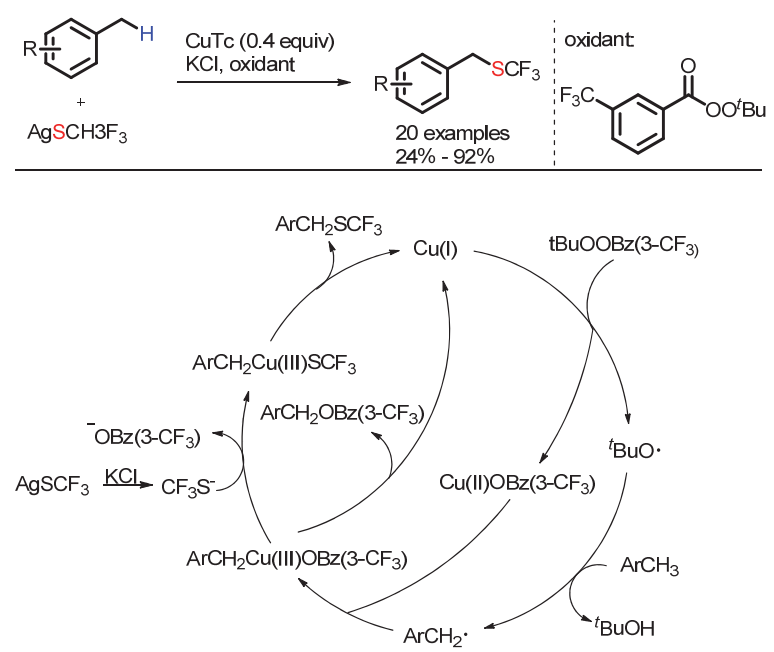

图 $8 \mathrm{Cu}$ 催化 $\mathrm{C}\left(s p^{3}\right)-\mathbf{H}$ 活化/三氟甲硫基化反应 57

Fig. $8 \mathrm{Cu}(\mathrm{II})$-catalyzed trifluoromethylthiolation via $\mathrm{C}\left(s p^{3}\right)-\mathrm{H}$ activation ${ }^{57}$.

Adapted from ACS publisher.

物。作者对此 $\mathrm{C}-\mathrm{H}$ 硫醚化反应提出了可能的反应 机理。

以上主要集中介绍 $\mathrm{C}\left(s p^{2}\right)-\mathrm{H}$ 和 $\mathrm{C}\left(s p^{3}\right)$ 官能化 构建硫醚, 对于 $\mathrm{C}(s p)-\mathrm{H}$ 的硫醚化反应也有报道。 2013年, 杨勇课题组 ${ }^{58}$ 报道了 $\mathrm{Cu}(\mathrm{I})$ 催化的端炔的 $\mathrm{C}-\mathrm{H}$ 官能化构建硫醚(图9)。该反应以氧气为氧化 剂, 硫醇或硫酚为硫源, 在温和条件下能以高收率 合成多种炔基芳基(烷基)硫醚, 但是该反应需用到 恶臭的硫酚或硫醇。

\section{$2.2 \mathrm{Pd}$ 催化 $\mathrm{C}-\mathrm{H}$ 官能化构建硫醚}

在过去的 20 年里, $\mathrm{Pd}$ 催化的 $\mathrm{C}-\mathrm{H}$ 官能化迅猛 发展 ${ }^{59-61}$, 合成化学家们也发展了一些 $\mathrm{C}-\mathrm{H}$ 官能 化构建硫醚的策略。

2014年, Nishihara等人 62 发展了一种邻位导向 $\mathrm{C}-\mathrm{H}$ 活化构建芳基硫醚的策略(图10)。该反应使

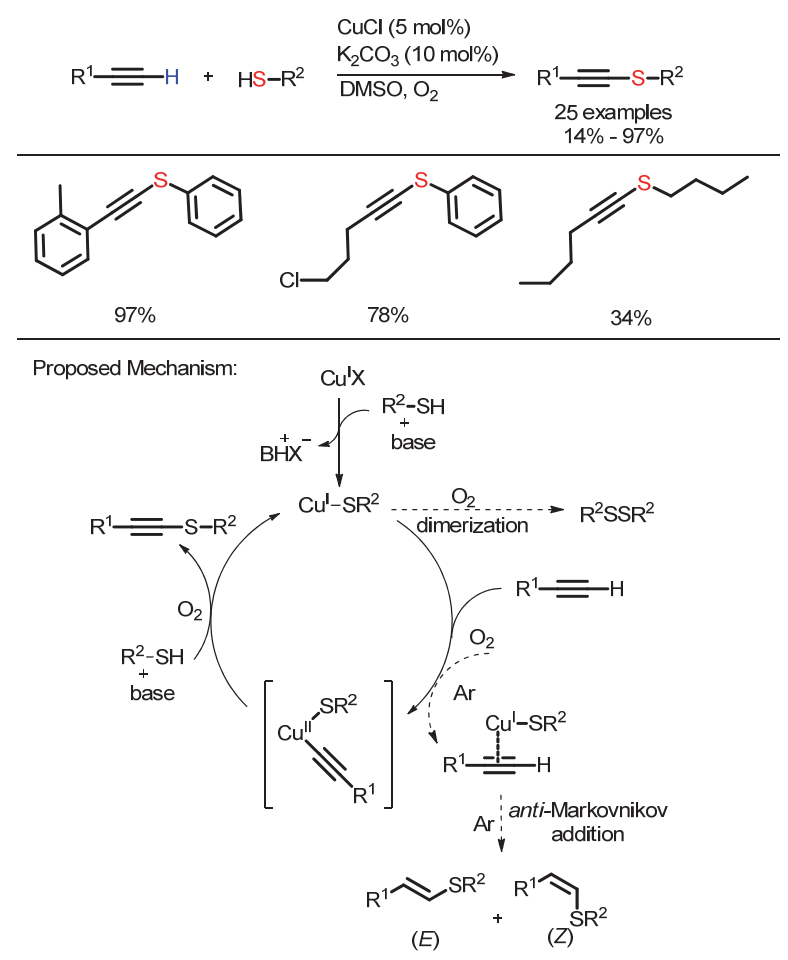

图 $9 \mathrm{Cu}(\mathrm{I})$ 催化 $\mathrm{C}(\mathrm{sp})-\mathrm{H}$ 硫醚化反应 ${ }^{58}$

Fig. $9 \mathrm{Cu}(\mathrm{I})$-catalyzed sulfenylation of $\mathrm{C}(s p)-\mathrm{H}$ bond ${ }^{58}$. Adapted from RSC publisher.

用 $\mathrm{PdCl}_{2}(\mathrm{NCPh})_{2}$ 为催化剂, $\mathrm{P}\left(2,4,6-\mathrm{Me}_{3} \mathrm{C}_{6} \mathrm{H}_{2}\right)_{3}$ 为配 体, $\mathrm{CuCl}_{2}$ 作为共催化剂, 使用二硫醚或硫醇为硫 源, 合成了一系列芳基硫醚。作者认为该反应中硫 醇在DMSO和铜盐的作用下转化为二硫醚作为反 应真正的硫醚化试剂，底物的选择及不同导向基 团的引入对邻位 $\mathrm{C}-\mathrm{H}$ 的单硫醚化或双硫醚化反 应具有重要影响, 机理研究表明该反应可能经历 $\mathrm{Pd}^{\mathrm{II}} / \mathrm{Pd}^{\mathrm{IV}}$ 的反应历程。

同年, Nishihara等人 ${ }^{63}$ 使用同样的 $\mathrm{C}-\mathrm{H}$ 官能 化策略, 以吡啶酰胺为导向基团合成了一系列 1菜胺的8位硫醚化产物, 作者认为该反应可能经历 一个五元环钯中间体。2015年，张兴国等人 ${ }^{64}$ 发展 了一种2-芳基吡啶的 $\mathrm{C}-\mathrm{H}$ 硫醚化反应, 该反应使 用 $N$-芳基颈基苯甲酰胺为硫源, 在无需外加氧化 剂条件下，通过调节硫源的当量可以调控单硫醚 化和双硫醚化产物的生成。

2014年, 沈其龙课题组 ${ }^{65}$ 报道了 Pd催化的邻位 导向的 $\mathrm{C}-\mathrm{H}$ 硫三氟甲基化反应, 当向底物中引入 吡啶等导向基团时, 使用 $N$-三氟甲硫基丁二酰亚 胺为硫源, 可以以中等到良好的收率、单一选择性 得到邻位单三氟甲硫基化产物。对于不同电性、带 有不同取代基的底物, 该策略表现出良好的普适 性。机理研究表明, 该反应可能经历 $\mathrm{Pd}(\mathrm{III})$ 或 $\mathrm{Pd}(\mathrm{IV})$ 中间体, 且芳基的 $\mathrm{C}-\mathrm{H}$ 活化可能不是该反 


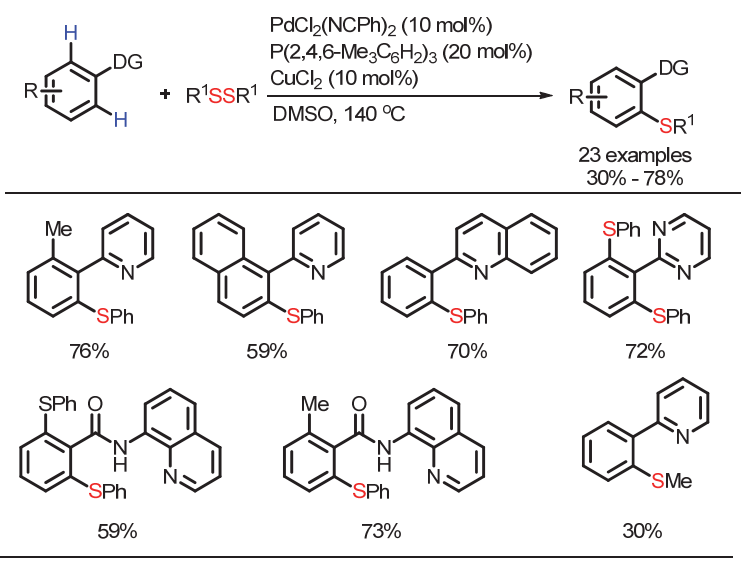

图 10 Pd 催化 2-苯基吡啶硫醚化反应 ${ }^{62}$

Fig. 10 Pd-catalyzed sulfenylation of 2-phenylpyridine ${ }^{62}$. Adapted from Wiley publisher.

应决速步。同年, 黄湧等人 ${ }^{66}$ 同样报道了 Pd催化2芳基吡啶的 $\mathrm{C}-\mathrm{H}$ 活化构建芳基三氟甲基硫醚的 反应。作者采用Selectedfluor为氧化剂, 采用配体 交换的策略发展了一种新的引入三氟甲硫基的方 法, 作者认为该反应可能也经历 $\mathrm{Pd}(\mathrm{II}) / \mathrm{Pd}(\mathrm{IV})$ 的催 化循环。

2015年, Besset等人 ${ }^{67}$ 实现了非活化 $s p^{3} \mathrm{C}-\mathrm{H}$ 的官能化(图11)。作者使用 8 -氨基喹啉为导向基 团, $\mathrm{PivOH}$ 为添加剂, 在亲电硫三氟甲基存在下, 高选择性的在酰胺 $\beta$ 位的 $s p^{3} \mathrm{C}-\mathrm{H}$ 实现了三氟甲硫 基化反应。

化学家们通过向底物中引入导向基团, 在 $\mathrm{Pd}$ 催化条件下实现了特定位点的 $\mathrm{C}-\mathrm{H}$ 官能化形成 硫醚键, 但是导向基团的引入势必会降低反应的 原子经济性和步骤经济性, 且部分导向基团难以 引入和脱除, 大大影响了底物的普适性。

近年来, 对于无导向基团的 $\mathrm{Pd}$ 催化 $\mathrm{C}-\mathrm{H}$ 官能 化构建硫醚化的反应化学家们也发展了一些方 法。2011年, Beller等人 ${ }^{68}$ 使用 $\mathrm{TsCN}$ 为硫源, 实现了 一系列富电子芳环的硫醚化反应, 但是该反应在底 物适用性上具有较大的局限。同年, Anbarasan等 人 ${ }^{69}$ 实现了 $\mathrm{Pd}$ 催化的非活化富电子芳烃的 $\mathrm{C}-\mathrm{H}$ 官 能化, 作者以 $N$-芳基巯基丁二酰亚胺为底物, 构建 了一系列非对称二芳基硫醚。

2015 年, Glorius等人 ${ }^{70}$ 发展了一种富电子杂芳 环 $\mathrm{C}-\mathrm{H}$ 硫醚化反应(图 12), 作者使用二芳基二硫 醚为硫源, 以商业可得的 $\mathrm{Pd} / \mathrm{Al}_{2} \mathrm{O}_{3}$ 为催化剂, 在当 量 $\mathrm{CuCl}_{2}$ 的作用下，合成了一系列非对称二芳基硫 醚, 作者还将该方法学应用于材料分子BTBT的合 成中。控制实验和机理研究表明, Pd和铜盐对该反 应均有重要作用, 且 $\mathrm{C}-\mathrm{H}$ 的断裂可能不是该反应 的决速步, 作者认为该反应决速步可能是反应中
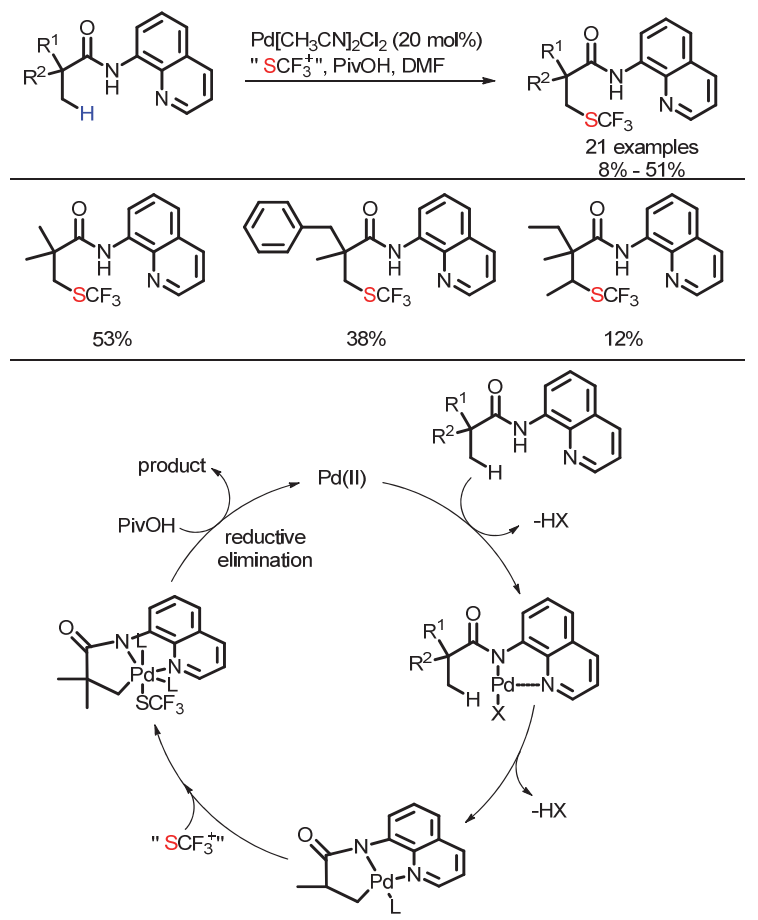

图 $11 P d$ 催化 $\mathrm{C}\left(s p^{3}\right)-\mathrm{H}$ 硫三氟甲基化反应 ${ }^{67}$

Fig. 11 Pd-catalyzed trifluoromethylthiolation of $\mathbf{C}\left(s p^{3}\right)-\mathrm{H}$ bonds ${ }^{67}$.

Adapted from ACS publisher.

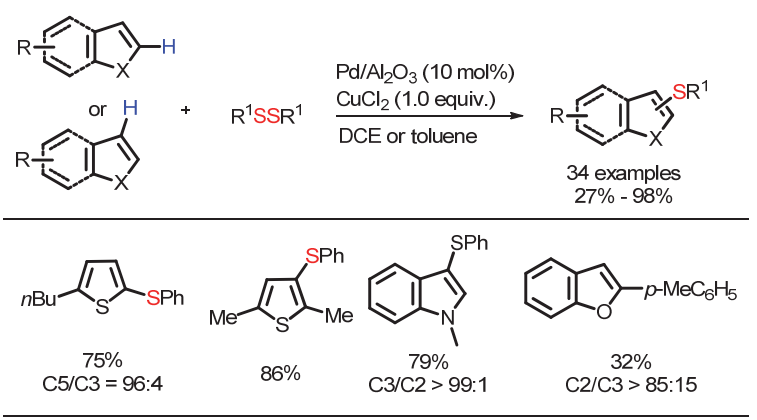

图 $12 P d$ 催化杂芳环硫醚化反应 ${ }^{70}$

Fig. 12 Pd-catalyzed sulfenylation of heteroarenes ${ }^{70}$.

Adapted from Wiley publisher.

生成的高亲电物种或者是活性催化物种对 $S-S$ 的 氧化加成。

2016年，Loh等人 ${ }^{71}$ 以烷基硫醇或芳基硫酚为 底物，成功实现了烯胺的 $\beta$ 位 $\mathrm{C}-\mathrm{H}$ 硫醚化反应。在 该反应中, 双膦配体 dppe的加入对防止催化剂毒 化有着重要作用, 醋酸铜和氧气在体系中起到共 氧化剂的作用, 该策略可以合成一系列的重要的 $\beta$ 氨基硫醚。

\section{$2.3 \mathrm{Ni}$ 催化 $\mathrm{C}-\mathrm{H}$ 官能化构建硫醚}

镍是一种廉价、储量丰富、低毒性的过渡金 属, 近年来镍催化的 $\mathrm{C}-\mathrm{H}$ 官能化受到越来越多的 化学家关注 ${ }^{72}$ 。2015年, 史炳峰课题组 ${ }^{73}$ 报道了邻 
位导向的 $\mathrm{C}-\mathrm{H}$ 活化构建二芳基硫醚(图 13)。作者 使用二芳基二硫醚为硫源, $\mathrm{NiCl}_{2} \cdot 6 \mathrm{H}_{2} \mathrm{O}$ 为催化剂, BINOL为配体以中等到良好的收率得到目标产 物。在同一时间, 陆红健 ${ }^{74}$ 、张玉红 ${ }^{75,76}$ 等人采用 同样的策略、使用类似的底物独立发表了镍催化 的 $s p^{2} \mathrm{C}-\mathrm{H}$ 的硫醚化反应。同年, 张玉红课题组 ${ }^{77}$ 报道了 $\mathrm{Ni}$ 催化的 $s p^{3} \mathrm{C}-\mathrm{H}$ 官能化反应, 作者使用 8氨基喹啉为导向基团, 用二芳基二硫醚为硫源, 在 $\mathrm{Ni}(\mathrm{OTf})_{2}$ 或 $\mathrm{Ni}(\mathrm{OAc})_{2}$ 的催化下成功活化羧酸衍生 物 $\beta$ 位 $\mathrm{C}-\mathrm{H}$, 构建一系列芳基烷基硫醚(图14)。几 乎同时, 邱仁华等人 ${ }^{78,79}$ 以同样使用 8 -氨基喹啉为 导向基, 以相似的策略也实现了 $s p^{3} \mathrm{C}-\mathrm{H}$ 的硫醚 化。

\section{$2.4 \mathrm{Rh}$ 催化 $\mathrm{C}-\mathrm{H}$ 官能化构建硫醚}

铑催化的 $\mathrm{C}-\mathrm{H}$ 官能化构建 $\mathrm{C}-\mathrm{S}$ 也是构建硫 醚的手段之一 ${ }^{80}$ 。2014年, 李援朝等人 ${ }^{81}$ 报道了 $\mathrm{Rh}$ 催化的 $\mathrm{C}\left(s p^{2}\right)-\mathrm{H}$ 的硫醚化反应, 对于不同的二烷 基硫醚或二芳基硫醚均可以以良好的收率得到目 标产物, 值得一提的是, 通过对反应条件的微调可 以实现产物生成单硫醚化产物或双硫醚化产物。

2015 年, 李兴伟等人 82 在吲哚 $\mathrm{N}$ 上引入导向基 团, 在 $\mathrm{Rh}$ 催化下成功实现了吲哚 2 位 $\mathrm{C}-\mathrm{H}$ 硫三氟 甲基化反应。作者做了一系列控制实验对反应机
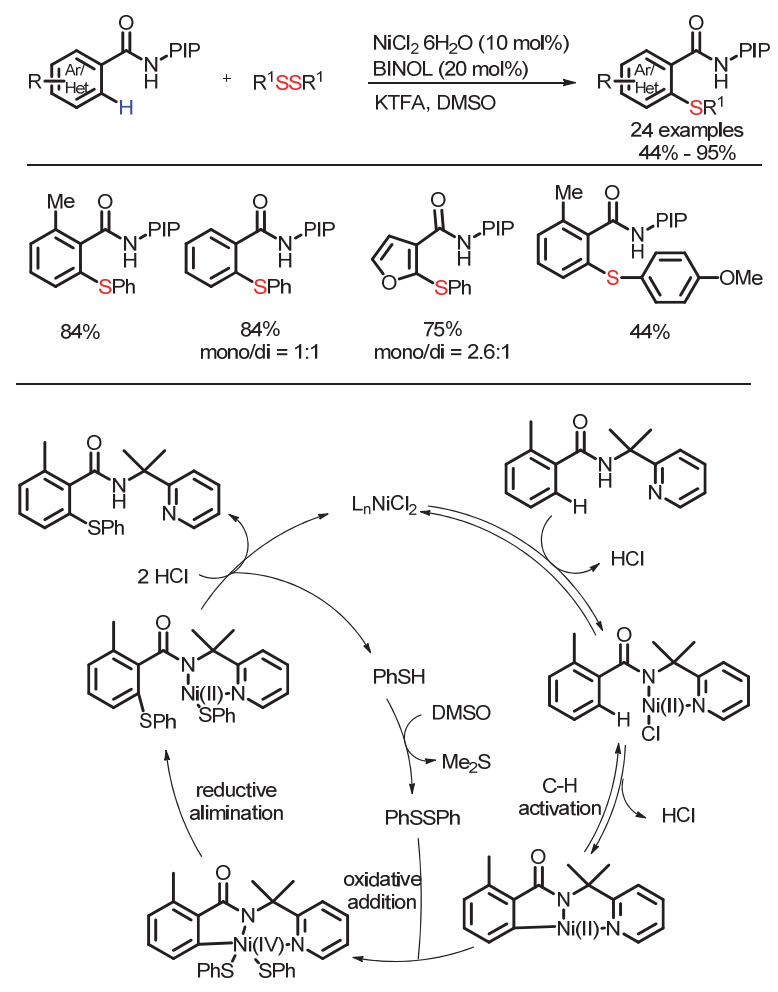

图 $13 \mathrm{Ni}$ 催化杂芳环硫醚化反应 ${ }^{73}$

Fig. 13 Ni-catalyzed sulfenylation of heteroarenes ${ }^{73}$.

Adapted from ASC publisher.

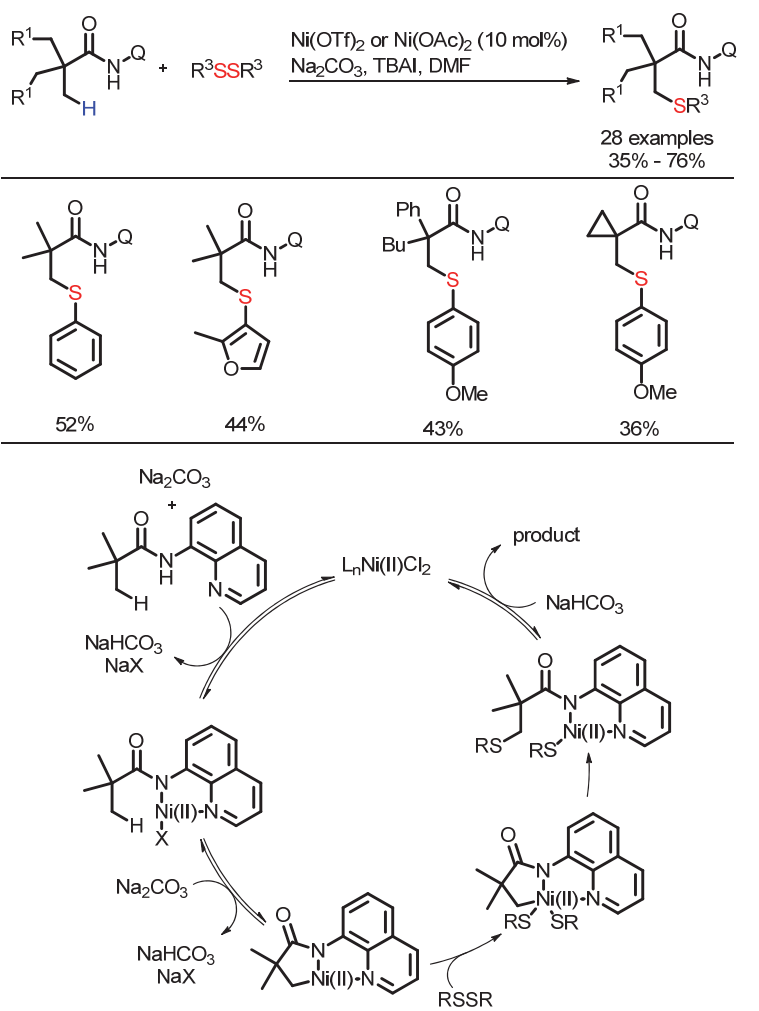

图 $14 \mathrm{Ni}$ 催化 $s p^{3} \mathrm{C}-\mathrm{H}$ 杂芳环硫醚化反应 77

Fig. 14 Ni-catalyzed sulfenylation of heteroarenes of $\mathbf{C}\left(s p^{3}\right)-\mathbf{H}$ bond ${ }^{77}$. Adapted from ACS publisher.

理进行探究发现富电子的吲哚更容易发生反应, 同 时 $\mathrm{C}-\mathrm{H}$ 活化是一个可逆过程且有可能是该反应 的决速步。2016, 王佰全等人 ${ }^{83}$ 在二氢吲哚 $\mathrm{N}$ 上引 入导向基团可以实现 $\mathrm{C} 7$ 位 $\mathrm{C}-\mathrm{H}$ 活化构建了一系 列二芳基硫醚。2017年, Samanta等人 ${ }^{84}$ 在吡啶 C3 位引入导向基团, 可以实现 $\mathrm{Rh}$ 催化的 $\mathrm{C} 4\left(s p^{2}\right)$ 和 $\mathrm{C} 2\left(s p^{3}\right)$ 的 $\mathrm{C}-\mathrm{H}$ 官能化(图 15), 在温和的反应条件 下，可以根据底物的不同合成二芳基硫醚或烷基芳基硫醚。

除了铜、钯、镍、铑等金属, 化学家们还发展 了一些其他过渡金属催化的 $\mathrm{C}-\mathrm{H}$ 官能化构建硫 醚 ${ }^{85-87}$ 。但是与上述介绍的几种过渡金属催化相 比, 其报道较少, 且催化模式和反应类型大体相 同，在此不做展开介绍。

\section{5 无金属催化的 $\mathbf{C}-\mathrm{H}$ 官能化构建硫醚}

近年来，无金属催化策略受到人们的广泛关 注，化学家们使用硫醇或硫酚 ${ }^{88-95}$ 、二硫醚96-105、 芳基磺酰氯 $106-109$ 、芳基磺酰胇 ${ }^{110-113}$ 、亚磺酸盐 114-121 等 ${ }^{122-129}$ 硫源在无金属催化的构建硫醚这一方向 已取得一些重要成果。其中不乏具有代表性的无 金属催化的 $\mathrm{C}-\mathrm{H}$ 官能化构建硫醚工作。

2015年，Penddinti等人 ${ }^{91}$ 使用碘作为催化剂、 

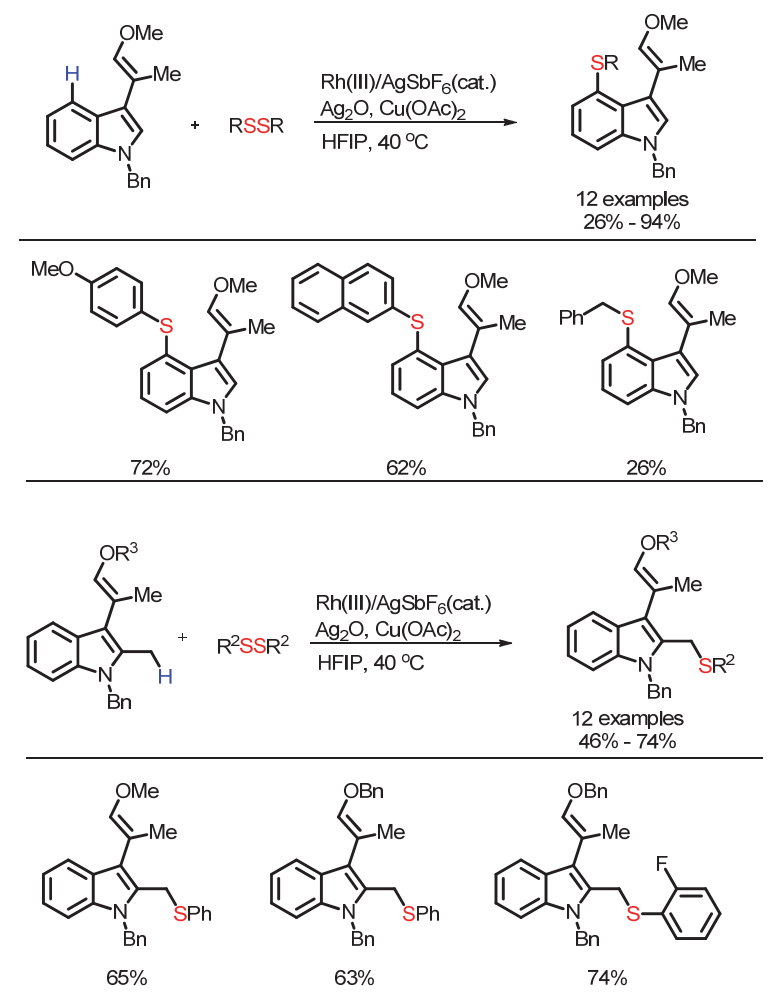

图 $15 R h$ 催化 $C\left(s p^{2}\right)-H$ 或 $C\left(s p^{3}\right)-H$ 官能化反应 ${ }^{84}$

Fig. 15 Rh-catalyzed $\mathrm{C}\left(s p^{2}\right)-\mathrm{H}$ or $\mathrm{C}\left(s p^{3}\right)-\mathrm{H}$ functionalizations ${ }^{84}$.

Adapted from ASC publisher.

无溶剂条件下的实现了富电子 $s p^{2} \mathrm{C}-\mathrm{H}$ 构建了一 系列二芳基硫醚和烯基芳基硫醚(图16), 该反应无 需过渡金属催化, 即可以高产率的得到相应的目 标产物, 且该反应可以放大至克级规模且产率没 有明显降低, 具有潜在的应用前景。作者提出了可 能的反应机理, 但是该反应只适用于富电子 $s p^{2}$ $\mathrm{C}-\mathrm{H}$ 的硫醚化反应。

2018年, 刘云云等人 ${ }^{94}$ 以 $\mathrm{KIO}_{3}$ 为催化剂使用芳 基硫醇为硫源合成了一系列吲哚3位取代的二芳 基硫醚(图17)。当用吲哚3位有取代基时, 可以得 到2位取代的硫醚化产物。一系列控制实验表明 二芳基硫醚可能不是反应活性中间体，作者在此 基础上提出了可能的反应机理: 首先, 异构化的吲 哚与 $\mathrm{KIO}_{3}$ 结合生成中间体 5 , 其互变异构的得到中 间体6并释放 $\mathrm{KOH}$ 得到中间体 7 。随后, 硫酚与中 间体 7 中碘氧双键加成中间体 8 , 其还原消除生成 产物并释放 $\mathrm{HIO}_{3}, \mathrm{HIO}_{3}$ 在 $\mathrm{KOH}$ 和 $\mathrm{O}_{2}$ 作用下再生催 化剂。

除了硫醇, 二硫醚也是一种常见的用于无金 属催化的硫源。在2011年, 向建南等人 ${ }^{96}$ 首次实现 了分子篮促进的氮原子邻位的 $s p^{3} \mathrm{C}-\mathrm{H}$ 官能化反 应(图18), 该反应无需过渡金属催化即可构建一系
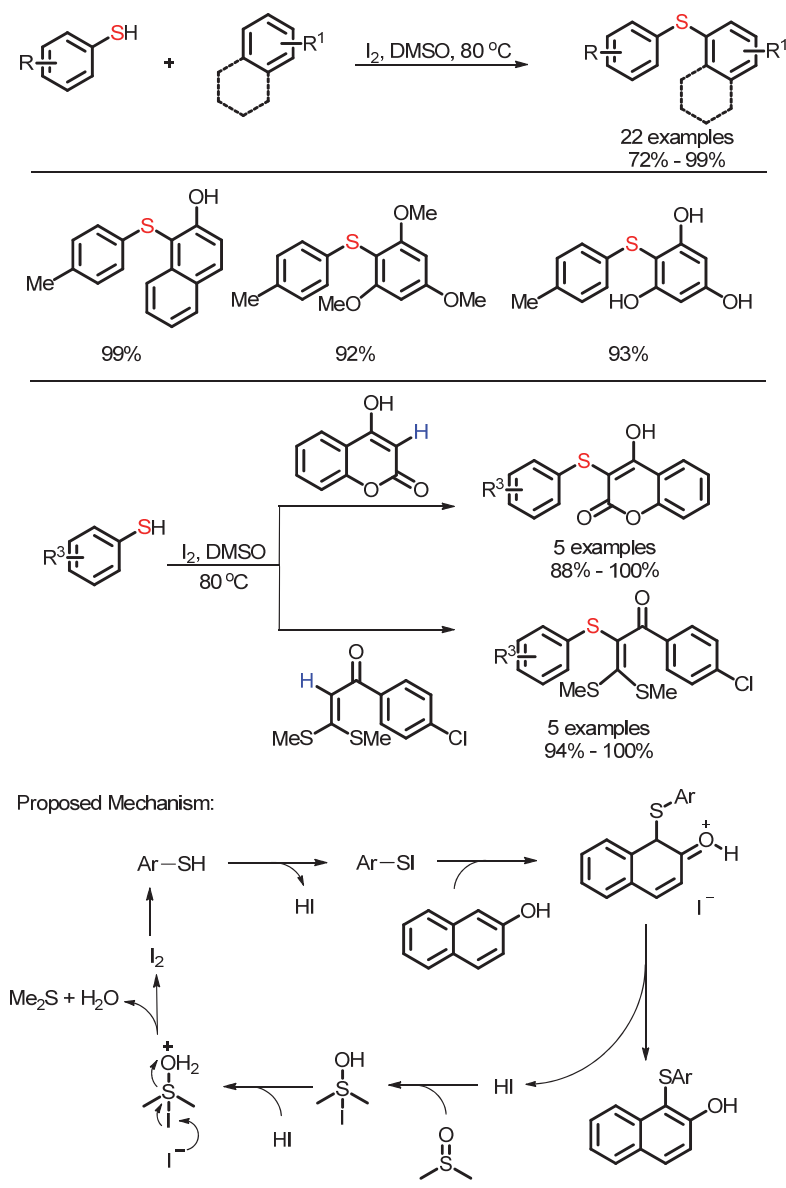

图 $16 I_{2}$ 催化 $\mathbf{C}\left(s p^{2}\right)-\mathbf{H}$ 硫醚化反应 ${ }^{91}$

Fig. $16 I_{2}$-catalyzed sulfenylation of $\mathrm{C}\left(s p^{2}\right)-H$ bond ${ }^{91}$.

Adapted from RSC publisher.

列含 $\mathrm{N} 、 \mathrm{~S}$ 双杂原子的硫醚化产物。该反应对于不 同的硫醚均能以良好到优秀的收率得到目标化合 物, 且对于 $\mathrm{N}$ 邻位的 $-\mathrm{CH}_{2}$ 与 $-\mathrm{CH}_{3}$ 相比具有更高 的反应活性。值得一提的是, 当使用邻氨基芳基二 硫醚时, 该反应可以进一步环合生成相应苯并噻 唑产物。但是该反应需以反应底物作为溶剂, 限制 了其进一步应用。2013年, 向建南等人 ${ }^{100}$ 以类似的 策略实现了醚类化合物氧邻位 $s p^{3} \mathrm{C}-\mathrm{H}$ 的硫醚化 反应。

使用二硫醚为硫源除了用于杂原子邻位 $s p^{3}$ $\mathrm{C}-\mathrm{H}$ 的硫醚化反应, 对于富电子杂芳环的 $\mathrm{C}-\mathrm{H}$ 硫

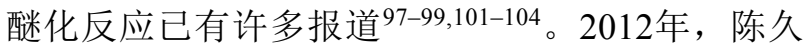
喜等人 98 使用二芳基二硫醚为硫源, 成功实现了 $N$ 澳代琥珀酰亚胺 $(\mathrm{NBS})$ 参与的吲哚 3 位 $\mathrm{C}-\mathrm{H}$ 官能 化合成了一系列二芳基硫醚(图 19), 该反应具有良 好的底物适用性, 吲哚上无论是连有吸电子还是 供电子基团均能以良好的收率得到目标产物。值 得一提的是, 当提高NBS的用量并升高温度, 可以 在吲哚的 2 位进一步溴化从而同时构建 $\mathrm{C}-\mathrm{S}$ 和 


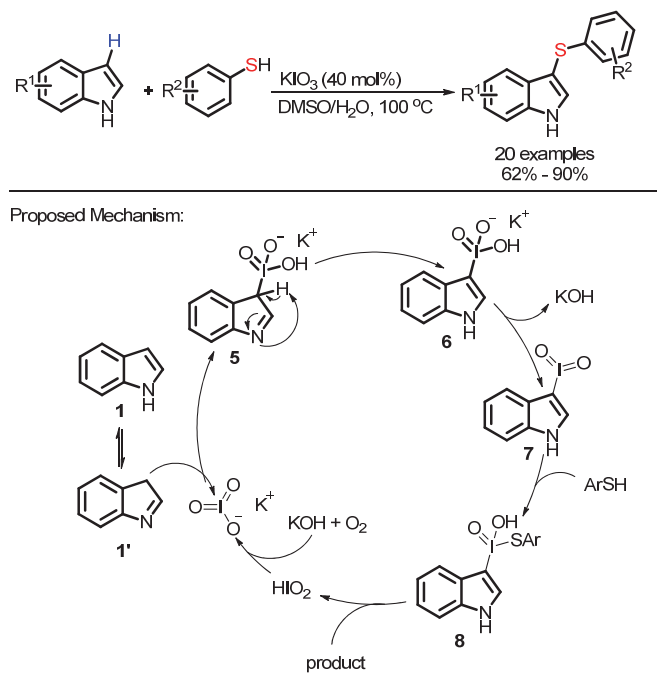

图 $17 \mathrm{KIO}_{3}$ 催化 $\mathrm{C}\left(s p^{2}\right)-\mathrm{H}$ 硫醚化反应 ${ }^{94}$

Fig. $17 \mathrm{KIO}_{3}$-catalyzed sulfenylation of

$$
\mathrm{C}\left(s p^{2}\right)-\mathrm{H} \text { bond }{ }^{94} \text {. }
$$

Adapted from Wiley publisher.
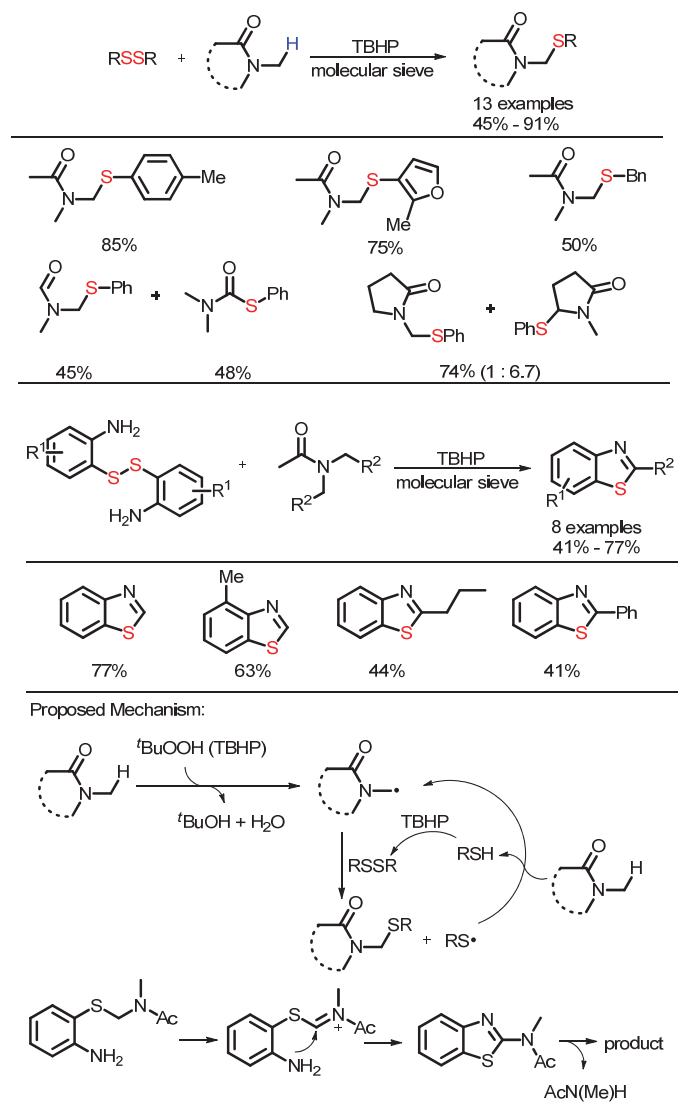

图 18 无金属催化 $\mathbf{C}\left(s p^{3}\right)-\mathbf{H}$ 硫醚化反应

Fig. 18 Sulfenylation of $\mathrm{C}\left(s p^{2}\right)-\mathrm{H}$ under metal-free conditions.

Adapted from ASC publisher ${ }^{96}$.

$\mathrm{C}-\mathrm{Br}$ 。机理研究表明, 芳基次磺酰溴可能是反应的 中间体，在此基础上，作者提出了可能的反应机理。

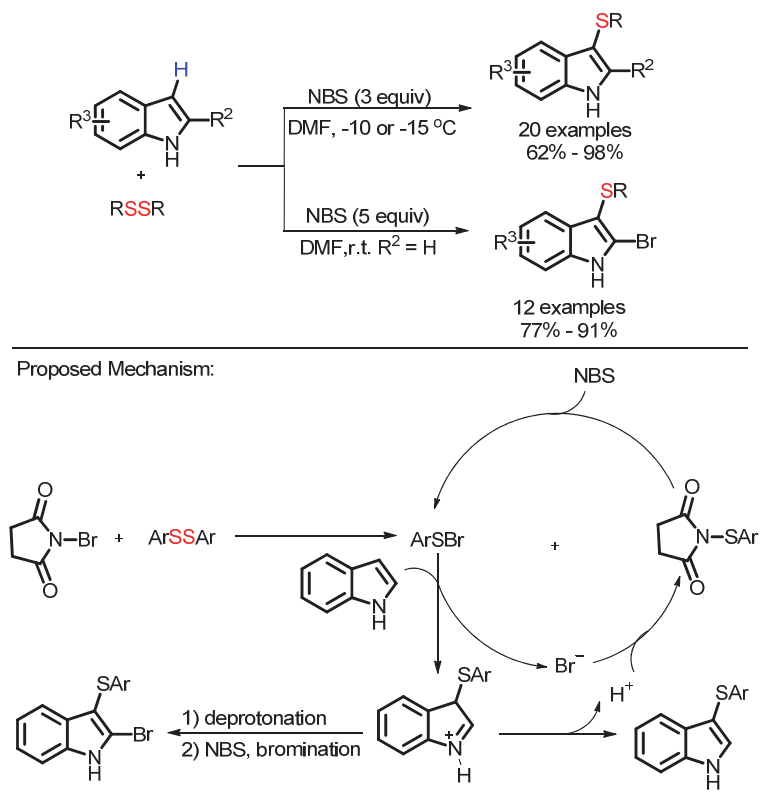

图 19 无金属催化硫醚化和溴化反应 ${ }^{98}$

Fig. 19 Metal-free sulfenylation and bromosulfenylation ${ }^{98}$. Adapted from Wiley publisher.

廉价易得的磺酰氯被广泛用作保护基、离去 基团及砜化试剂。2011年, 游劲松等人 ${ }^{106}$ 将其作为 硫源首次应用于无金属催化的构建硫醚的反应中 (图20)，该反应使用甲苯为溶剂，在三苯基膦存在 条件下成功实现了富电子芳环或杂芳环 $\mathrm{C}-\mathrm{H}$ 的
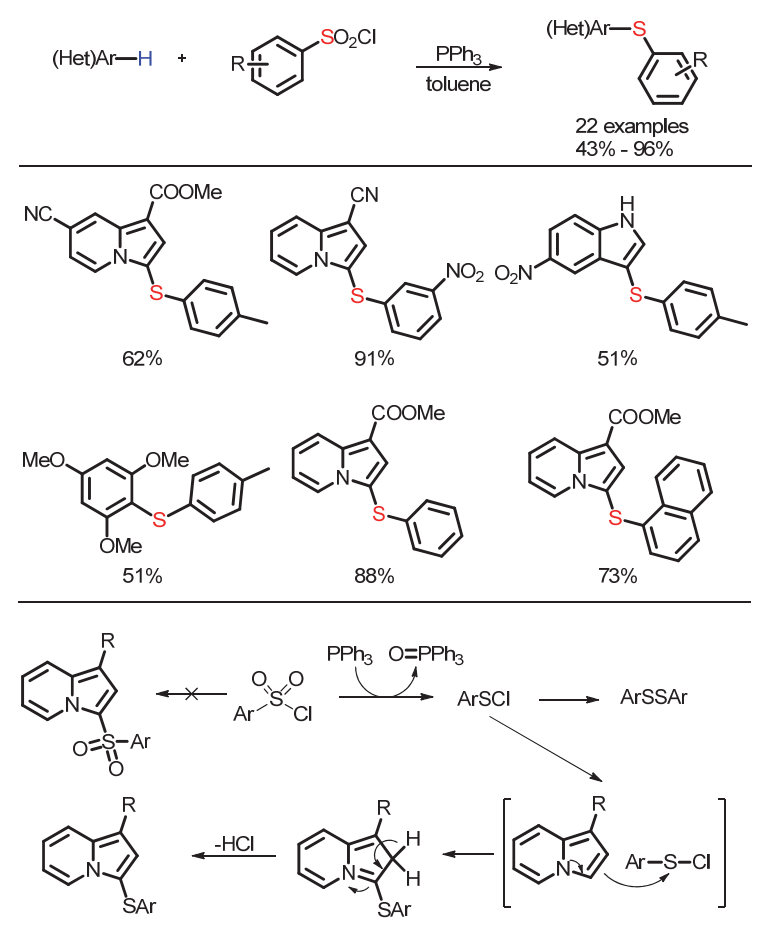

图 20 以芳基磺酰氯为硫源的硫醚化反应 106

Fig. 20 Sulfenylation using arylsulfonyl chlorides ${ }^{106}$. Adapted from ASC publisher. 
官能化。该策略具有较好的底物普适性, 对于吸电 子和富电子的芳基磺酰氯均能以良好的收率得到 目标产物。作者认为该反应可能经历以下历程: 首 先芳基磺酰氯在三苯基膦的还原下生成活性中间 体芳基次磺酰氯, 随后芳基次磺酰氯与富电子芳环 反应并脱去氯化氢得到目标产物。

除了芳基磺酰氯之外, 2016年, 易文斌等人 109 使用含氟磺酰氯为硫源, 在亚磷酸二乙酯的作用 下, 成功合成了一系列含氟烷基硫醚化合物。该反 应具有良好的底物适用性, 对于富电子芳环能以 良好到优秀的收率和优异的选择性得到目标产 物, 且对于不同的含氟烷基磺酰氯(比如 $\mathrm{CF}_{3} \mathrm{SO}_{2} \mathrm{Cl}$ 、 $\mathrm{C}_{4} \mathrm{~F}_{9} \mathrm{SO}_{2} \mathrm{Cl} 、 \mathrm{C}_{8} \mathrm{~F}_{17} \mathrm{SO}_{2} \mathrm{Cl} 、 \mathrm{CF}_{2} \mathrm{HS}_{2} \mathrm{Cl} 、 \mathrm{CH}_{2} \mathrm{CF}_{3} \mathrm{SO}_{2} \mathrm{Cl}$ ) 均能较好地适用于该体系, 具有潜在的应用前景。 作者认为该反应机理可能与前面所述机理类似形 成次磺酰氯中间体。

磺酰肼也可以作为硫源应用于无金属催化的 $\mathrm{C}-\mathrm{H}$ 官能化中, 2013年, 田仕凯等人 110 使用磺酰 肼为硫源, $\mathrm{I}_{2}$ 为催化剂成功实现了吲哚 3 位 $\mathrm{C}-\mathrm{H}$ 的 硫醚化反应, 当吲哚的 3 位被取代时, 该硫醚化反 应可成功发生在吲哚的2位(图21)。研究表明催化 量的 $\mathrm{I}_{2}$ 的加入可以极大加速磺酰肼的分解, 使其作 为一种高效的硫醚化反应的试剂成为可能, 其反 应机理可能为碘催化下生成次磺酰碘与富电子吲 哚高区域选择性的发生傅克反应得到目标产物。

在无金属催化的 $\mathrm{C}-\mathrm{H}$ 官能化构建硫醚这一方 向, 亚磺酸盐相较于磺酰肼报道更多。其反应机制 可能为亚磺酸盐在碘单质和还原剂作用下, 或者 在碘盐的作用下生成次磺酰碘与富电子芳环发生
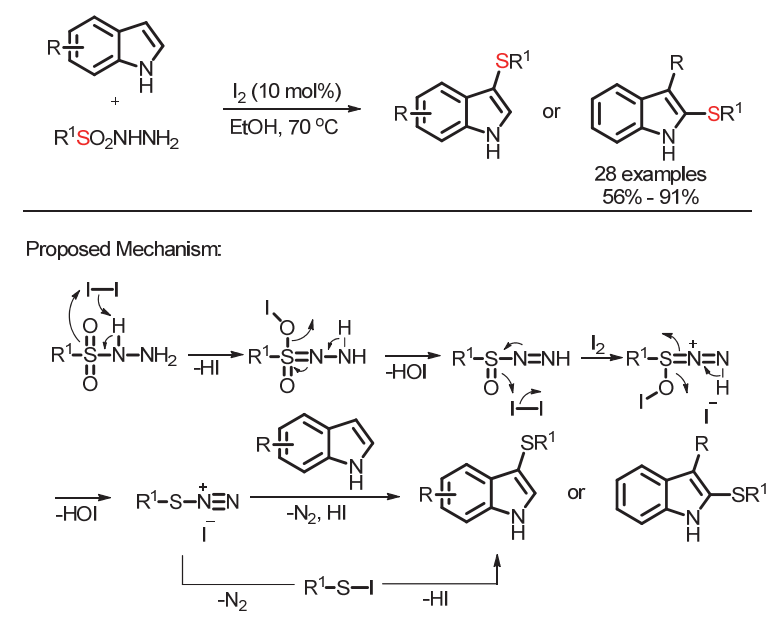

$\mathrm{HI}+\mathrm{HOI} \longrightarrow \mathrm{H}_{2} \mathrm{O}+\mathrm{I}_{2}$

图 $21 I_{2}$ 催化的吲哚的硫醚化反应 ${ }^{110}$

Fig. $21 I_{2}$-catalyzed sulfenylation of indoles ${ }^{110}$. Adapted from Wiley publisher.
傅克反应 ${ }^{115-117,121}$, 但是其报道底物与以上反应类 似主要集中在吲哚等富电子杂芳环。在2016年, 易 文斌课题组 ${ }^{119}$ 以芳基亚磺酸钠为硫源, 在 $\mathrm{I}_{2}$ 和 $\mathrm{PPh}_{3}$ 的作用下实现了芳胺、酚、酮的 $\mathrm{C}-\mathrm{H}$ 官能化反应 (图22), 该反应体系对于未保护的芳胺、酚羟基的 敏感基团能很好兼容, 且反应具有很好的区域选 择性。值得一提的是当芳胺上连有吸电子基团以 及在复杂体系中该反应仍能以令人满意的产率发 生。2018年, 易文斌课题组 ${ }^{124}$ 同样使用无臭的芳基 亚磺酸钠为硫源, 在水相 $/ \mathrm{PPh}_{3}$ 体系中合成一系列 炔硫醚，作者提出了可能的反应机理认为芳基亚 磺酸钠在体系中生成活性中间体硫碘化合物, 其 与芳基炔烃发生加成随后在强碱作用下脱除一分 子碘化氢即可得到目标产物。

除了上述介绍的几种硫源之外, 亚磺酸 ${ }^{125,129}$ 、 Bunte盐 ${ }^{126}$ 、亚磺酸酯 ${ }^{127,130}$ 也可被用于无金属催化 的 $\mathrm{C}-\mathrm{H}$ 硫醚化反应, 但是其底物仍集中在富电子
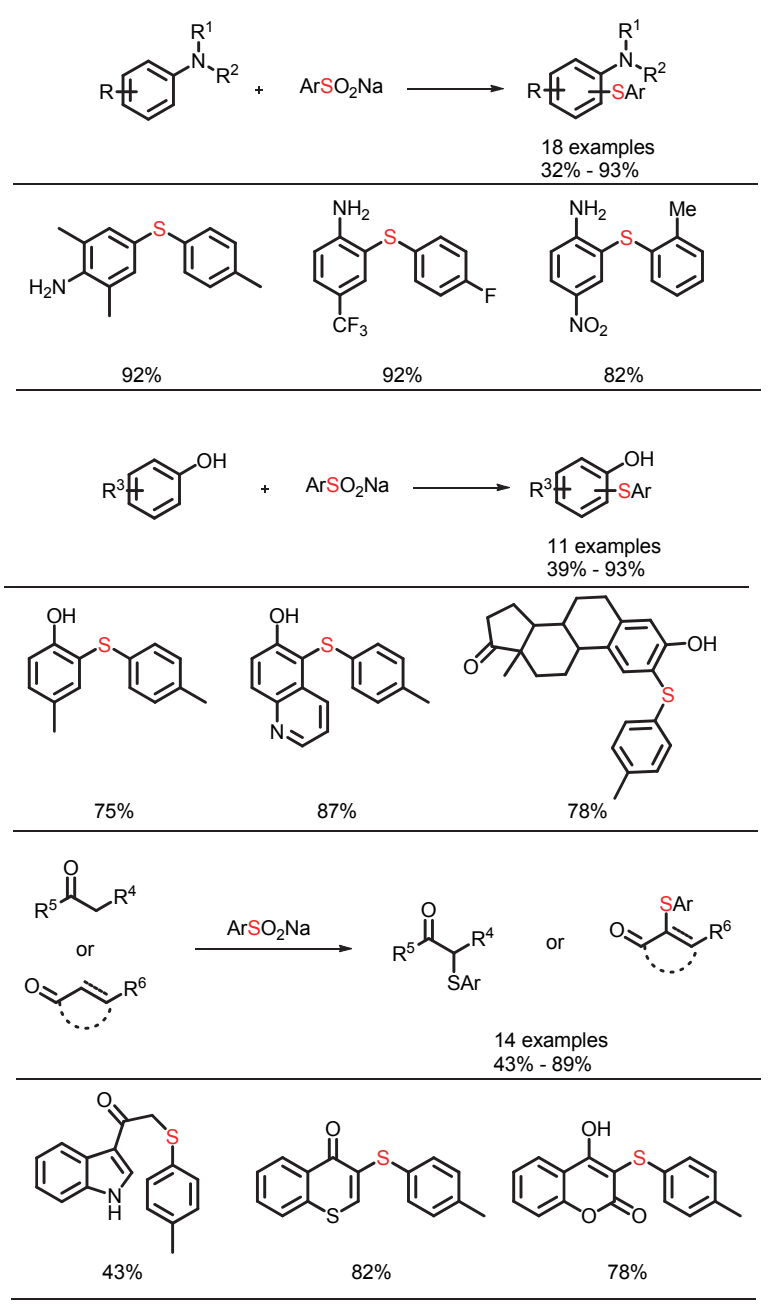

图 $22 I_{2}$ 参与硫醚化反应 119

Fig. 22 I2-mediated sulfenylation ${ }^{119}$. Adapted from Wiley publisher. 
Directing groups:<smiles>[Y]c1cccc2cccnc12</smiles><smiles>CC(C)(N)c1ccccn1</smiles><smiles>Cc1ccccn1</smiles>

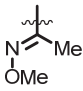

图 23 本文主要导向基团的种类

Fig. 23 Species in directing groups.

的杂芳环或者需要反应底物为溶剂, 在此不做展 开介绍。

\section{3 结论}

本文阐述了近年来过渡金属催化/参与或无过 渡金属催化的 $\mathrm{C}-\mathrm{H}$ 官能化构建硫醚这一方向的 研究进展。在部分过渡金属催化的 $\mathrm{C}-\mathrm{H}$ 硫醚化反 应中, 导向基团的引入对于 $\mathrm{C}-\mathrm{H}$ 官能化有着重要 的作用，本文介绍的导向基团主要为 8 -氨基喹啉、 吡啶等含氮导向基团(图23), 向体系中引入导向基 可以实现特定位置 $\mathrm{C}-\mathrm{H}$ 的官能化反应, 提高其化 学选择性的同时带来了导向基团脱除困难、原子 经济性降低的问题。对于无导向基体系, 其 $\mathrm{C}-\mathrm{H}$ 硫醚化反应主要发生在富电子杂芳环的活性位 点、富电子芳环中苯环 $\mathrm{C}-\mathrm{H}$ 、芐位等高活性位点, 底物局限性较大。

因此，尽管化学家们发展了一系列构建硫醚 的策略, 但是对于 $\mathrm{C}-\mathrm{H}$ 官能化构建硫醚这一方兴 未艾的领域, 仍然存在许多亟待解决的问题。比 如, 1)在官能化构建硫醚这一方向, $s p^{3} \mathrm{C}-\mathrm{H}$ 比 $s p^{2}$ $\mathrm{C}-\mathrm{H}$ 更难活化且相应报道较少; 2)为了活化特定 位置的 $\mathrm{C}-\mathrm{H}$ 常需向底物中引入导向基团, 且其导 向基团大多活化邻位 $\mathrm{C}-\mathrm{H}$ 。因此, 发展更多新的 配体和新的催化模式实现间位、对位的 $\mathrm{C}-\mathrm{H}$ 硫醚 化是一个重要的发展方向。而且需要发展更多以 底物中已有的羧基、酯基、氨基、羟基等为导向基 团而无需特地引入其他导向基团, 或者发展更多 无导向基团的 $\mathrm{C}-\mathrm{H}$ 官能化反应构建硫醚；3)目前 仍未见以 $\mathrm{C}-\mathrm{H}$ 官能化构建不对称 $\mathrm{C}-\mathrm{S}$ 的报道, 因 此需发展更多手性配体以及新的催化模式构建手 性硫醚；4)目前, C-H官能化构建硫醚这一方向 机理尚不明确, 因此, 更多的机理探究用以探明反 应机理并在此基础上设计出新的催化体系和反应 模式尤为重要。

\section{References}

(1) Ilardi, E. A.; Vitaku, E.; Njardarson, J. T. J. Med. Chem. 2014, 57, 2832. doi: $10.1021 / \mathrm{jm} 401375 \mathrm{q}$

(2) Smith, B. R.; Eastman, C. M.; Njardarson, J. T. J. Med. Chem. 2014, 57, 9764. doi: $10.1021 / \mathrm{jm} 501105 \mathrm{n}$
(3) Feng, M.; Tang, B.; Liang, S.; Jiang, X. Curr. Top. Med. Chem. 2016, 16, 1200. doi: 10.2174/1568026615666150915111741

(4) Xiong, H. -Y.; Pannecoucke, X.; Besset, T. Chem. -Eur. J. 2016, 22, 16734. doi: 10.1002/chem.201603438

(5) Mishra, A.; Ma, C. Q.; Bäuerle, P. Chem. Rev. 2009, 109, 1141. doi: $10.1021 /$ cr8004229

(6) Wang, M.; Fan, Q.; Jiang, X. Org. Lett. 2016, 18, 5756. doi: 10.1021/acs.orglett.6b03078

(7) Borthwick, A. D. Chem. Rev. 2012, 112, 3641 doi: $10.1021 / \mathrm{cr} 200398 \mathrm{y}$

(8) Nicolaou, K. C.; Hale, C. R. H.; Nilewski, C.; Ioannidou, H. A. Chem. Soc. Rev. 2012, 41, 5185. doi: 10.1039/C2CS35116A

(9) Davison, E. K.; Sperry, J. J. Nat. Prod. 2017, 80, 3060 doi: 10.1021/acs.jnatprod.7b00575

(10) Liu, H.; Jiang, X. Chem. Asian J. 2013, 8, 2546. doi: 10.1002/asia.201300636

(11) Nair, D. P.; Podgorski, M.; Shunsuke, C.; Gong, T.; Xi, W. X.; Fenoli, C. R.; Bowman, C. N. Chem. Mater. 2014, 26, 724 doi: $10.1021 / \mathrm{cm} 402180 \mathrm{t}$

(12) Bürchstümmer, H.; Weissenstein, A.; Bilalas. D.; Würthner, F. J. Org. Chem. 2011, 76, 2426. doi: 10.1021/jo2003117

(13) Takimiya, K.; Osaka, I.; Mori, T.; Nakano, M. Acc. Chem. Res. 2014, 47, 1493. doi: 10.1021/ar400282g

(14) Nakazawa, T.; Xu, J.; Nishikawa, T.; Oda, T.; Fujita, A.; Ukai, K.; Mangindaan, R. E. P.; Rotinsulu, H.; Kobayashi, H.; Namikoshi, M. J. Nat. Prod. 2007, 70, 439. doi: 10.1021/np060593c

(15) Oda, T.; Fujiwara, T.; Liu, H.; Ukai, K.; Mangindaan, R. E. P.; Mochizuki, M.; Namikoshi, M. Marine Drugs 2006, 4, 15. doi: $10.3390 / \mathrm{md} 401015$

(16) Thase, M. E.; Macfadden, W.; Weisler, R. H.; Chang, W.; Paulsson, B.; Khan, A.; Calabrese, J. R. J. Clin. Psychopharmacol. 2006, 26, 600. doi: 10.1097/01.jcp.0000248603.76231.b7

(17) Chen,S.; Wang, M.; Jiang, X. Chin. J. Chem. 2018, 36, 921. doi: $10.1002 /$ cjoc. 201800242

(18) Lin, D. Y.; Zhang, S. -Z.; Block, E.; Katz, L. C. Nature 2005, 434, 470. doi: $10.1038 /$ nature 03414

(19) Kondo, T.; Mitsudo, T. -A. Chem. Rev. 2000, 100, 3205. doi: $10.1021 / \operatorname{cr} 9902749$

(20) Beletskaya, I. P.; Anikov, V. P. Chem. Rev. 2011, 111, 1596. doi: $10.1021 / \mathrm{cr} 100347 \mathrm{k}$

(21) Alvaro, E.; Hartwig, J. F. J. Am. Chem. Soc. 2009, 131, 7858. doi: $10.1021 /$ ja901793w

(22) Fernandez-Rodriguez, M. A.; Shen, Q.; Hartwig, J. F. J. Am. Chem. Soc. 2006, 128, 2180. doi :10.1021/ja0580340

(23) Ma, D.; Geng, Q.; Zhang, H.; Jiang, Y. Angew. Chem. Int. Ed. 2010, 49, 1291. doi: 10.1002/anie.200905646 
(24) Cao, H.; Chen, L.; Liu, J.; Cai, H.; Deng, H.; Chen, G.; Yan, C.; Chen, Y. RSC Adv. 2015, 5, 22356. doi: 10.1039/C5RA01342A

(25) Ferraccioli, R. Cur. Org. Synth. 2012, 9, 96. doi: $10.2174 / 157017912798889224$

(26) Liao, Y.; Peng, Y.; Qi, H.; Deng, G. -J.; Gong, H.; Li, C. -J. Chem. Commun. 2015, 51, 1031. doi: 10.1039/C4CC08370A

(27) Chen, X.; Hao, X. -S.; Goodhue, C. E.; Yu, J. -Q. J. Am. Chem. Soc. 2006, 128, 6790. doi: 10.1021/ja061715q

(28) Chu, L.; Yue, X.; Qing, F. -L. Org. Lett. 2010, 12, 1644. doi: $10.1021 / \mathrm{ol} 100449 \mathrm{c}$

(29) Fukuzawa, S. -I.; Shimizu, E.; Atsumi, Y.; Haga, M.; Ogata, K. Tetrahedron Lett. 2009, 50, 2374. doi : 10.1016/j.tetlet.2009.02.214

(30) Zhang, S.; Qian, P.; Zhang, M.; Hu, M.; Cheng, J. J. Org. Chem. 2010, 75, 6732. doi: 10.1021/jo1014849

(31) Zhou, A.-X.; Liu, X.-Y.; Yang, K.; Zhao, S. -C.; Liang, Y. -M. Org. Biomol. Chem. 2011, 9, 5456. doi: 10.1039/C1OB05395G

(32) Ranjit, S.; Lee, R.; Heryadi, D.; Shen, C.; Wu, J.; Zhang, P.; Huang, K.-W.; Liu, X. J. Org. Chem. 2011, 76, 8999. doi: $10.1021 /$ jo2017444

(33) Alves, D.; Lara, R. G.; Contreira, M. E.; Radatz, C. S.; Duarte, L. F. B.; Perin, G. Tetrahedron Lett. 2012, 53, 3364. doi: $10.1016 /$ j.tetlet.2012.04.094

(34) Dai, C.; Xu. Z.; Huang, F.; Yu, Z.; Gao, Y. -F. J. Org. Chem. 2012, 77, 4414. doi: $10.1021 /$ jo202624s

(35) Rosario, A. R.; Casola, K. K.; Oliveira, C. E. S.; Zeni, G. Adv. Synth. Catal. 2013, 355, 2960. doi: 10.1002/adsc.201300497

(36) Zheng, Z.; Qi, D.; Shi, L. Catal. Commun. 2015, 66, 83. doi: 10.1016/j.catcom.2015.03.023

(37) Leroux, F.; Jeschke, P.; Schlosser, M. Chem. Rev. 2005, 105, 827. doi: $10.1021 / \mathrm{cr} 040075 \mathrm{~b}$

(38) Boiko, V. N. Beilstein J. Org. Chem. 2010, 6, 880. doi: $10.3762 /$ bjoc. 6.88

(39) Jeschke, P. Pest Manag. Sci. 2010, 66, 10. doi: 10.1002/ps.1829

(40) Yang, Y. -D.; Azuma, A.; Tokunaga, E.; Yamasaki, M.; Shiro, M.; Shibata, N. J. Am. Chem. Soc. 2013, 135, 8782. doi: $10.1021 / \mathrm{ja} 402455 \mathrm{f}$

(41) Shao, X.; Wang, X.; Yang, T.; Lu, L.; Shen, Q. Angew. Chem. Int. Ed. 2013, 52, 3457. doi: 10.1002/anie.201209817

(42) Li, Y.; Ye, Z.; Bellman, T. M.; Chi, T.; Dai, M. Org. Lett. 2015, 17, 2186. doi: 10.1021 /acs.orglett.5b00782

(43) Hu, F.; Shao, X.; Zhu, D.; Lu, L.; Shen, Q. Angew. Chem. Int. Ed. 2014, 53, 6105. doi: 10.1002/anie.201402573

(44) Bootwicha, T.; Liu, X.; Pluta, R.; Atodiresei, I, Rueping, M. Angew. Chem. Int. Ed. 2013, 52, 12856. doi: 10.1002/anie.201304957

(45) Baert, F.; Colomb, J.; Billard, T. Angew. Chem. Int. Ed. 2012, 51, 10382. doi: $10.1002 /$ anie. 201205156
(46) Kang, K.; Xu, C.; Shen, Q. Org. Chem. Front. 2014, 1, 294. doi: $10.1039 / \mathrm{C} 3 \mathrm{QO} 00068 \mathrm{~K}$

(47) Liu, J.; Chu, L.; Qing, F.-L. Org. Lett. 2013, 15, 894. doi: $10.1021 / \mathrm{ol} 400032 \mathrm{~g}$

(48) Wang, Q.; Qi, Z.; Xie, F.; Li, X. Adv. Synth. Catal. 2015, 357, 355. doi: $10.1002 /$ adsc. 201400717

(49) Yin, G.; Kalvet, I.; Schoenebeck, F. Angew. Chem. Int. Ed. 2015, 54, 6809. doi: 10.1002/anie.201501617

(50) Zhang, C. -P.; Vicic, D. A. J. Am. Chem. Soc. 2012, 134, 183. doi: $10.1021 / \mathrm{ja} 210364 \mathrm{r}$

(51) Zhu, X. -L.; Xu, J. -H.; Cheng, D. -J.; Zhao, L. -J.; Liu, X. -Y.; Tan, B. Org. Lett. 2014, 16, 2192. doi: 10.1021/o15006888

(52) Zhu, L.; Wang, G.; Gou, Q.; Xu, Z.; Zhang, D.; Wang, R. Org. Lett. 2014, 16, 5390. doi: 10.1021/ol502624z

(53) Ye, K. -Y.; Zhang, X.; Dai, L. -X.; You, S. -L. J. Org. Chem. 2014, 79, 12106. doi: 10.1021/jo5019393

(54) Tran, L. D.; Popov, I.; Daugulis, O. J. Am. Chem. Soc. 2012, 134, 18237. doi: $10.1021 /$ ja3092278

(55) Yan, X. -B.; Gao, P.; Yang, H. -B.; Li, Y. -X.; Liu, X. -Y.; Liang, Y. -M. Tetrahedron 2014, 70, 8730. doi : 10.1016/j.tet.2014.09.042

(56) Shibahara, F.; Kanai, T.; Yamaguchi, E.; Kamei, A.; Yamauchi, T.; Murai, T. Chem. Asian J. 2014, 9, 237. doi: 10.1002/asia.201300882

(57) Chen, C.; Xu, X. -H.; Yang, B.; Qing, F. -L. Org. Lett. 2014, 16, 3372. doi: $10.1021 / \mathrm{ol} 501400 \mathrm{u}$

(58) Yang, Y.; Dong, W.; Gou, Y.; Rioux, R. M. Green Chem. 2013, 15, 3170. doi: 10.1039/C3GC41330F

(59) Lyons, T. W.; Sanford, M. S. Chem. Rev. 2010, 110, 1147. doi: $10.1021 / \mathrm{cr} 900184 \mathrm{e}$

(60) Daugulis, O.; Do, H. -Q.; Shabashov, D. Acc. Chem. Res. 2009, 42, 1074. doi: $10.1021 / \mathrm{ar} 9000058$

(61) He, J.; Wasa, M.; Chan, K. S. L.; Shao, Q.; Yu, J. -Q. Chem. Rev. 2017, 117, 8754. doi: 10.1021/acs.chemrev.6b00622

(62) Iwasaki, M.; Iyanaga, M.; Tsuchiya, Y.; Nishimura, Y.; Li, W.; Li, Z.; Nishihara, Y. Chem. Eur. J. 2014, 20, 2459. doi: $10.1002 /$ chem. 201304717

(63) Iwasaki, M.; Kaneshika, W.; Tsushiya, Y.; Nakajima, K.; Nishihara, Y. J. Org. Chem. 2014, 79, 11330. doi: 10.1021/jo502274t

(64) Zhang, X. -S.; Li, G.; Zhang, X. -G.; Zhang, X. -H. Tetrahedron 2015, 71, 5458. doi: 10.1016/j.tet.2015.06.077

(65) Xu, C.; Shen, Q. Org. Lett. 2014, 16, 2046. doi: 10.1021/o15006533

(66) Yin, W.; Wang, Z.; Huang, Y. Adv. Synth. Catal. 2014, 356, 2998. doi: $10.1002 /$ adsc. 201400362

(67) Xiong, H. -Y.; Besset, T.; Cahard, D.; Pannecoucke, X. J. Org. Chem. 2015, 80, 4204. doi: 10.1021/acs.joc.5b00505

(68) Anbarasan, P.; Neumann, H.; Beller, M. Chem. Commun. 2011, 47, 3233. doi: $10.1039 / \mathrm{C} 0 \mathrm{CC} 04405 \mathrm{~A}$ 
(69) Saravanan, P.; Anbarasan, P. Org. Lett. 2014, 16, 848. doi: 10.1021/o14036209

(70) Vásquez-Céspedes, S.; Ferry, A.; Candish, L.; Glorius, F. Angew. Chem. Int. Ed. 2015, 54, 5772. doi: 10.1002/anie.201411997

(71) Jiang, Y.; Liang, G.; Zhang, C.; Lohn, T. -P. Eur. J. Org. Chem. 2016, 3326. doi: 10.1002/ejoc. 201600588

(72) Nakao, Y. Chem. Rec. 2011, 11, 242. doi: 10.1002/tcr.201100023

(73) Yan, S. -Y.; Liu, Y. -J.; Liu, B.; Liu, Y. -H.; Shi, B. -F. Chem. Commun. 2015, 51, 4069. doi: 10.1039/C4CC10446C

(74) Yang, K.; Wang, Y.; Chen, X.; Kadi, A. A.; Fun, H. -K.; Sun, H.; Zhang, Y.; Lu, H. Chem. Commun. 2015, 51, 3582. doi: 10.1039/C4CC10431E

(75) Lin, C.; Li, D.; Wang, B.; Yao, J.; Zhang, Y. Org. Lett. 2015, 17, 1328. doi: 10.1021/acs.orglett.5b00337

(76) Reddy, V. P.; Qiu, R.; Iwasaki, T.; Kambe, N. Org. Biomol. Chem. 2015, 13, 6803. doi: 10.1039/C5OB00149H

(77) Lin, C.; Yu, W.; Yao, J.; Wang, B.; Liu, Z.; Zhang, Y. Org. Lett. 2015, 17, 1340. doi: 10.1021/acs.orglett.5b00471

(78) Wang, X.; Qiu, R.; Yan, C.; Reddy, V. P.; Zhu, L.; Xu, X.; Yin, S. -F. Org. Lett. 2015, 17, 1970. doi: 10.1021/acs.orglett.5b00706

(79) Ye, X.; Petersen, J. L.; Shi, X. Chem. Commun. 2015, 51, 7863. doi: 10.1039/C5CC01970B

(80) Shen, C.; Zhang, P.; Sun, Q.; Bai, S.; Hor, T. S. A.; Liu, X. Chem. Soc. Rev. 2015, 44, 291. doi: 10.1039/C4CS00239C

(81) Yang, Y.; Hou, W.; Qin, L.; Du, J.; Feng, H.; Zhou, B.; Li, Y. Chem. Eur. J. 2014, 20, 416. doi: 10.1002/chem.201303730

(82) Wang, Q.; Xie, F.; Li, X. J. Org. Chem. 2015, 80, 8361. doi: 10.1021/acs.joc.5b00940

(83) Xie, W.; Li, B.; Wang, B. J. Org. Chem. 2016, 81, 396. doi: 10.1021/acs.joc.5b01943

(84) Maity, S. Karmakar, U.; Samanta, R. Chem. Commun. 2017, 53, 12197. doi: 10.1039/C7CC07086A

(85) Gensch, T.; Klauck, F. J. R.; Glorius, F. Angew. Chem. Int. Ed. 2016, 55, 11287. doi: 10.1002/anie.201605193

(86) Mandal, A.; Dana, S.; Sahoo, H.; Grandhi, G. S.; Baidya, M. Org. Lett. 2017, 19, 2430. doi: 10.1021/acs.orglett.7b00996

(87) Zhang, M.; Zhang, S.; Pan, C.; Chen, F. Synth. Commun. 2012, 42, 2844. doi: 10.1080/00397911.2011.569867

(88) Ravi, C.; Mohan, D. C.; Adimurthy, S. Org. Lett. 2014, 16, 2978. doi: $10.1021 / 01501117 \mathrm{z}$

(89) Liu, Y.; Zhang, Y.; Hu, C.; Wan, J. -P.; Wen, C. RSC Adv. 2014, 4, 35528. doi: 10.1039/C4RA05206D

(90) Hiebel, M. -A.; Raboin, S. B. Green Chem. 2015, 17, 937. doi: $10.1039 / \mathrm{C} 4 \mathrm{GC} 01462 \mathrm{~F}$

(91) Parumala, S. K. R.; Peddinti, R.; K. Green Chem. 2015, 17, 4068. doi: 10.1039/C5GC00403A
(92) Yang, D.; Sun, P.; Wei, W.; Meng, L.; He, L.; Fang, B.; Jiang, W.; Wang, H. Org. Chem. Front. 2016, 3, 1457.

doi: 10.1039/C6Q000407E

(93) Chouldhury, P.; Roy, B.; Basu, B. Asian J. Org. Chem. 2017, 6, 1569. doi: 10.1002/ajoc. 201700275

(94) Bai, F.; Zhang, S.; Wei, L.; Liu, Y. Asian J. Org. Chem. 2018, 7, 371. doi: 10.1002/ajoc. 201700677

(95) Li, B.; Chen, Z.; Cao, H.; Zhao, H. Org. Lett. 2018, 20, 3291. doi: 10.1021/acs.orglett.8b01168

(96) Tang, R. -Y.; Xie, Y. -X.; Xie, Y. -L.; Xiang, J. -N.; Li, J. -H. Chem. Commun. 2011, 47, 12867. doi: 10.1039/C1CC15397H

(97) Zou, L. -H.; Reball, J.; Mottweiler, J.; Bolm, C. Chem. Commun. 2012, 48, 11307. doi: 10.1039/C2CC36711D

(98) Huang, D.; Chen, J.; Dan, W.; Ding, J.; Liu, M.; Wu, H. Adv. Synth. Catal. 2012, 354, 2123. doi: 10.1002/adsc.201200227

(99) Ge, W.; Wei, Y. Green Chem. 2012, 14, 2066. doi: $10.1039 / \mathrm{C} 2 \mathrm{GC} 35337 \mathrm{G}$

(100) Guo, S. -R.; Yuan, Y. -Q.; Xiang, J. -N. Org. Lett. 2013, 15, 4654. doi: $10.1021 / \mathrm{ol} 402281 \mathrm{f}$

(101) Sang, P.; Chen, Z.; Zou, J.; Zhang, Y. Green Chem. 2013, 15, 2096. doi: $10.1039 / \mathrm{C} 3 \mathrm{GC} 40724 \mathrm{~A}$

(102) Azeredo, J.; Godoi, M.; Martins, G. M.; Silverira, C. C.; Braga, A. L. J. Org. Chem. 2014, 79, 4125. doi: 10.1021/jo5000779

(103) Gao, Z.; Zhu, X.; Zhang, R. RSC Adv. 2014, 4, 19891. doi: 10.1039/C4RA01240B

(104) Rafique, J.; Saba, S.; Franco, M. S.; Bettanin, L.; Schneider, A. R.; Silva, L. T.; Braga, A. L. Chem. Eur. J. 2018, 24, 4173. doi: 10.1002/chem.201705404

(105) Yu, Y.; Zhou, Y.; Song, Z.; Liang, G. Org. Biomol. Chem. 2018, 16, 4958. doi: 10.1039/C8OB00948A

(106) Wu, Q.; Zhao, D.; Qin, X.; Lan, J.; You, J. Chem. Commun. 2011, 47, 9188. doi: 10.1039/C1CC13633J

(107) Kumaraswamy, G.; Raju, R.; Narayanarao, V. RSC Adv. 2015, 5, 22718. doi: 10.1039/C5RA00646E

(108) Zhao, W.; Zhou, A. ChemCatChem 2015, 7, 3464. doi: $10.1002 /$ cctc. 201500673

(109) Jiang, L.; Yi, W.; Liu, Q. Adv. Synth. Catal. 2016, 358, 3700. doi: $10.1002 /$ adsc. 201600651

(110) Yang, F. -L.; Tian, S. -K. Angew. Chem. Int. Ed. 2013, 52, 4929. doi: 10.1002/anie. 201301437

(111) Kang, X.; Yan, R.; Yu, G.; Pang, X.; Liu, X.; Li, X.; Xiang, L.; Huang, G. J. Org. Chem. 2014, 79, 10605. doi: 10.1021/jo501778h

(112) Bagdi, A. K.; Mitra, S.; Ghosh, M.; Hajra, A. Org. Biomol. Chem. 2015, 13, 3314. doi: 10.1039/C5OB00033E

(113) Zhao, W.; Xie, P.; Bian, Z.; Zhou, A.; Ge, H.; Zhang, M.; Ding, Y.; Zheng, L. J. Org. Chem. 2015, 80, 9167. 
doi: 10.1021/acs.joc.5b01602

(114) Rao, H.; Wang, P.; Wang, J.; Li, Z.; Sun, X.; Cao, S. RSC Adv. 2014, 4, 49165. doi: 10.1039/C4RA08669D

(115) Xiao, F.; Xie, H.; Liu, S.; Deng, G. -J. Adv. Synth. Catal. 2014, 356, 364. doi: $10.1002 /$ adsc. 201300773

(116) Ding, Y.; Wu, W.; Zhao, W.; Li, Y.; Xie, P.; Huang, Y.; Liu, Y.; Zhou, A. Org. Biomol. Chem. 2016, 14, 1428. doi: $10.1039 / \mathrm{C} 5 \mathrm{OB} 02073 \mathrm{E}$

(117) Huang, X.; Wang, S.; Li, B.; Wang, X.; Ge, Z.; Li, R. RSC Adv. 2015, 5, 22654. doi: 10.1039/C4RA17237J

(118) Wang, D.; Zhang, R.; Lin, S.; Yan, Z.; Guo, S. RSC Adv. 2015, 5, 108030. doi: $10.1039 / C 5 R A 24351 C$

(119) Lin, Y. -M.; Lu, G. -P.; Wang, G. -X.; Yi, W. -B. Adv. Synth. Catal. 2016, 358, 4100. doi: 10.1002/adsc.201600846

(120) Gou, Y. -J.; Lu, S.; Tian, L. -L.; Huang, E. -L.; Hao, X. -Q.; Zhu, X.; Shao, T.; Song, M. -P. J. Org. Chem. 2018, 83, 338. doi: $10.1021 /$ acs.joc. $7 \mathrm{~b} 02734$

(121) Liu, C.; Fan, J.; Wu, M.; Chen, J.; Xie, M. Chin. J. Chem. 2018, 36, 819. doi: 10.1002/cjoc.201800164

(122) Tudge, M.; Tamiya, M.; Savarin, C.; Humphrey, G. Org. Lett. 2006,
8, 565. doi: $10.1021 / \mathrm{ol} 052615 \mathrm{c}$

(123) Marcantoni, E.; Cipolletti, R.; Marsili, L.; Menichetti, S.; Properzi, R.; Viglianisi, C. Eur. J. Org. Chem. 2013, 132. doi: 10.1002/ejoc.201201100

(124) Lin, Y.; Yi, W. Chin. J. Org. Chem. 2018, 38, 1207. [林雅玫, 易文 斌. 有机化学, 2018, 38, 1207]. doi: 10.6023/cjoc201711036

(125) Liu, C.; Ding, L. -H. Org. Biomol. Chem. 2015, 13, 2251. doi: $10.1039 / \mathrm{C} 4 \mathrm{OB} 02575 \mathrm{~J}$

(126) Qi, H.; Zhang, T.; Wan, K.; Lou, M. J. Org. Chem. 2016, 81, 4262. doi: $10.1021 /$ acs.joc.6b00636

(127) Li, Y.; Zhu, F.; Wang, Z.; Wu, X. -F. Chem. Asian J. 2016, 11, 3503. doi: 10.1002/asia.201601376

(128) Fan, W.; Yang, Z.; Jiang, B.; Li, G. Org. Chem. Front. 2017, 4, 1091. doi: $10.1039 / \mathrm{C} 6 \mathrm{QO} 00851 \mathrm{H}$

(129) Sun, P.; Yang, D.; Wei, W.; Jiang, M.; Wang, Z.; Zhang, L.; Zhang, H.; Zhang, Z.; Wang, Y.; Wang, H. Green Chem. 2017, 19, 4785. doi: $10.1039 / \mathrm{C} 7 \mathrm{GC} 01891 \mathrm{~F}$

(130) Yang, X.; Bao, Y.; Dai, Z.; Zhou, Q.; Yang, F. Green Chem. 2018, 20, 3727. doi: $10.1039 / \mathrm{C} 8 \mathrm{GC} 01764 \mathrm{~F}$ 